\title{
Complex-scaled infinite elements for resonance problems in heterogeneous open systems
}

\section{Lothar Nannen $^{1}$ (D) . Markus Wess ${ }^{1,2}$}

Received: 8 September 2020 / Accepted: 22 December 2021 / Published online: 9 February 2022 (C) The Author(s) 2022

\begin{abstract}
The technique of complex scaling for time harmonic wave-type equations relies on a complex coordinate stretching to generate exponentially decaying solutions. In this work, we use a Galerkin method with ansatz functions with infinite support to discretize complex-scaled scalar Helmholtz-type resonance problems with inhomogeneous exterior domains. We show super-algebraic convergence of the method with respect to the number of unknowns in radial direction. Numerical examples underline the theoretical findings.
\end{abstract}

Keywords Helmholtz-type resonance problems · Complex scaling . Infinite elements · Heterogeneous exterior domains

\section{Introduction}

Perfectly matched layers (PMLs) are a popular method for treating acoustic resonance and scattering problems in open domains (cf. [3, 6, 21] or [13, 24] for the same method under the name complex scaling). The idea behind this method is the application of a complex coordinate stretching to the unbounded exterior domain to generate exponentially decaying outgoing solutions. Subsequently, the exterior domain is truncated to a bounded layer resulting in a bounded computational domain. The truncated problem on the now bounded domain can be discretized using a

\footnotetext{
Communicated by: Ivan Graham

Lothar Nannen

lothar.nannen@tuwien.ac.at

Markus Wess

markus.wess@ensta-paris.fr

1 Institute for Analysis and Scientific Computing of TU Wien, Vienna, Austria

2 POEMS, CNRS, INRIA, ENSTA Paris, Institut Polytechnique de Paris, Palaiseau, France
} 
standard finite element method. Complex scaling (i.e., the choice of the exterior domain and the complex coordinate stretching) can be done in various ways: parallel to the coordinate axes, resulting in so-called cartesian scalings (cf. [5]), in radial direction (cf. [6]), or in normal direction with respect to a convex interface (cf. [23]). In this work we will focus on radial scalings, although the method can be extended to cartesian or normal scalings in a straightforward way (cf. Remark 4.1).

PMLs are rather easy to implement in standard finite element codes but have the downside that there are many method parameters to choose: the scaling function, the thickness of the layer, and the finite element discretization of the layer. All these method parameters have to be balanced to ensure the efficiency of the method. The parameter choice becomes even more delicate if there are inhomogeneities such as potentials in the exterior domain.

In this work we present and analyze a method which is also based on complex scaling but omits the truncation of the exterior domain. In this way we simplify the choice of method parameters. Another idea to omit truncation was introduced in [4], where singular scaling profiles in combination with standard finite elements are used. In contrast, we use a linear scaling profile combined with non-standard basis functions. Convergence of our method is established based on the abstract results of [18, 19] for holomorphic Fredholm operator functions.

As radial basis functions we choose generalized Laguerre functions leading to the complex-scaled infinite elements. They converge super-algebraically with respect to the number of radial unknowns $N$, i.e., the error decays faster than any polynomial in $1 / N$. Moreover, they lead to sparse and well-conditioned discretization matrices, and are simple to couple to interior problems. Note that a standard PML would lead to an exponentially decreasing truncation error. Nevertheless, if a finite element discretization with piecewise polynomials is used in the perfectly matched layer, the total error will decrease merely polynomially with respect to $1 / N$ due to the finite element error. It turns out that, for homogeneous exterior problems, the complex-scaled infinite elements are equivalent to the Hardy space infinite elements introduced in [14].

The remainder of the paper is organized as follows: In Section 2 we define the complex-scaled problems in question and give a brief explanation of the method of complex scaling. In Section 3 we explain the used tensor product exterior discretizations. The complex-scaled infinite elements are defined in Section 4. Section 5 contains the convergence analysis. The main results are summarized in Section 5.4. The numerical experiments of Section 6 underline our theoretical findings. The numerical tests also include a performance study of the infinite elements compared to a conventional radial PML. We end with a short conclusion.

\section{Problem setting}

Since we are concerned with Helmholtz-type problems on unbounded domains we start by specifying the domains in question. Afterwards we define the radial complex scalings we use, followed by a short motivation of the method for homogeneous and inhomogeneous exterior problems. The weak formulation of the complex-scaled problem under investigation is stated in Problem 2.1. 


\subsection{Complex coordinate stretching}

Let $\Omega \subset \mathbb{R}^{3}$ be an unbounded open domain such that $\Omega$ can be decomposed into a bounded interior part $\Omega_{\text {int }}$ with Lipschitz boundary $\partial \Omega_{\text {int }}$, an unbounded exterior part $\Omega_{\text {ext }}$, and an interface $\Gamma$. The open sets $\Omega_{\text {int }}, \Omega_{\text {ext }}, \Gamma \Omega_{\text {int }}, \Omega_{\text {ext }}$ and the interface $\Gamma$ should fulfill the following assumptions:

(i) $\Omega=\Omega_{\text {int }} \dot{\cup} \Gamma \dot{\cup} \Omega_{\text {ext }}$, where $\dot{U}$ denotes the union of two disjoint sets,

(ii) there exists $R>0$, such that $\Omega_{\text {int }}=\Omega \cap B_{R}(0), \Omega_{\text {ext }}=\Omega \backslash \overline{\Omega_{\text {int }}}$ and $\Gamma=\{\mathbf{x} \in \Omega:\|x\|=R\}$, and

(iii) $\Omega_{\mathrm{ext}}=\left\{\left(1+\frac{\xi}{R}\right) \hat{\mathbf{x}}: \hat{\mathbf{x}} \in \Gamma, \xi \in \mathbb{R}_{>0}\right\}$.

Note that these conditions imply that for each $\mathbf{x} \in \Omega_{\text {ext }} \cup \Gamma$ there exists a unique pair $(\xi, \hat{\mathbf{x}}) \in \mathbb{R}_{\geq 0} \times \Gamma$, such that

$$
\mathbf{x}=\left(1+\frac{\xi}{R}\right) \hat{\mathrm{x}}
$$

For the mapping defined by (2.1) we also write $\mathbf{x}(\xi, \hat{x})$ and $(\xi(\mathbf{x}), \hat{x}(\mathbf{x}))$ for the inverse mapping. Figure 1 illustrates the cross section of two examples of the setting described above.

In this work we consider linear complex scalings of the form

$$
\gamma(\mathbf{x}):= \begin{cases}\mathbf{x}, & \mathbf{x} \in \Omega_{\text {int }}, \\ \left(1+\frac{\sigma \xi(\mathbf{x})}{R}\right) \hat{\mathrm{x}}(\mathbf{x}), & \mathbf{x} \in \Omega_{\mathrm{ext}},\end{cases}
$$

for a given parameter $\sigma \in \mathbb{C} \backslash\{0\}$. Note that although we define the scaling for general non-zero parameters $\sigma$ we will assume positive real and imaginary part for $\sigma$ later on. We denote the Jacobian of the scaling $\gamma$ by

$$
J_{\sigma}(\mathbf{x})=J_{\sigma}\left(\left(x_{1}, x_{2}, x_{3}\right)^{T}\right):=\left(\frac{\partial \gamma_{i}\left(\left(x_{1}, x_{2}, x_{3}\right)^{T}\right)}{\partial x_{j}}\right)_{i=1,2,3, j=1,2,3} .
$$

Note that $J_{\sigma}$ is smooth on $\Omega_{\text {int }}$ and $\Omega_{\text {ext }}$ respectively.
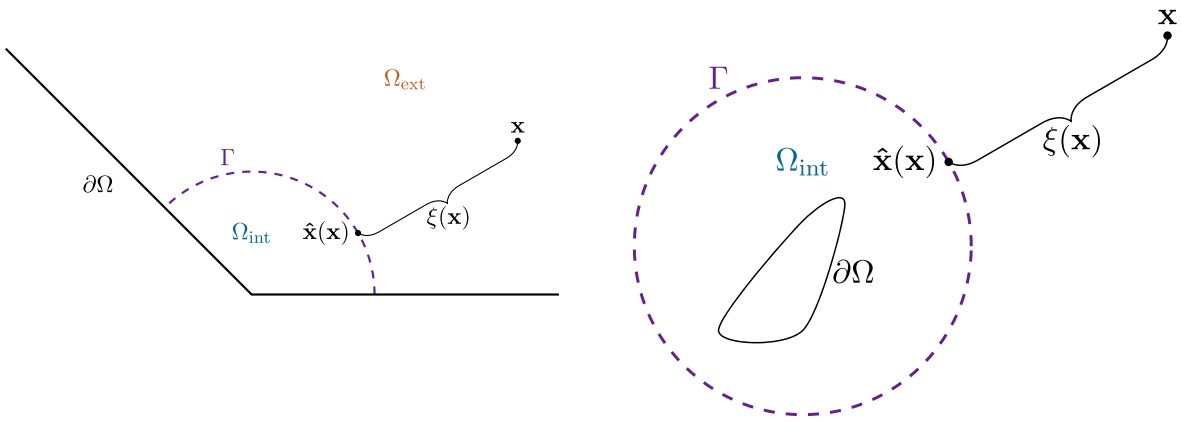

Fig. 1 Cross sections of three-dimensional example domains and exterior coordinates 


\subsection{The complex-scaled problem for homogeneous exterior domains}

To motivate the use of the complex-scaled problem for inhomogeneous exterior domains, we use this subsection to sketch the derivation of this technique in the case of homogeneous exterior domains (cf. [6, 10, 21]).

A version of the classical Helmholtz eigenvalue problem is to find an eigenpair $\omega \in \mathbb{C}, u \in H_{\text {loc }}^{2}(\Omega) \backslash\{0\}$, such that

$$
-\Delta u(\mathbf{x})=\omega^{2} p(\mathbf{x}) u(\mathbf{x}), \quad \mathbf{x} \in \Omega,
$$

with boundary conditions on $\partial \Omega$, a suitable potential function $p \in L^{\infty}(\Omega)$ with $p(\mathbf{x}) \equiv 1$ for $\mathbf{x} \in \Omega_{\mathrm{ext}}$, and a radiation condition for $u$. For resonance problems this radiation condition can be specified by imposing that in $\Omega_{\text {ext }}$ the eigenfunctions $u$ are linear combinations of functions which are spherical Hankel functions of the first kind (with respect to $\xi$ ) and spherical harmonics (with respect to $\hat{x}$ ).

Since the spherical Hankel functions are analytic we may consider the analytic continuation of an eigenfunction $u$ to the complex-scaled domain $\gamma(\Omega)$. Due to the asymptotic behavior of the spherical Hankel functions of the first kind the function $w:=u \circ \gamma$ is exponentially decreasing in $\Omega_{\text {ext }}$ with respect to $\xi$ and therefore an element of $H^{1}\left(\Omega_{\text {ext }}\right)$ as long as $\Im(\sigma \omega)>0$.

Thus (assuming homogeneous Neumann boundary conditions) the pair $\omega, w$ satisfies the complex-scaled weak formulation

$$
\begin{aligned}
\int_{\Omega} J_{\sigma}(\mathbf{x})^{-T} \nabla w(\mathbf{x}) \cdot J_{\sigma}(\mathbf{x})^{-T} \overline{\nabla v(\mathbf{x})} & \operatorname{det} J_{\sigma}(\mathbf{x}) d \mathbf{x} \\
& =\omega^{2} \int_{\Omega} p(\mathbf{x}) w(\mathbf{x}) \overline{v(\mathbf{x})} \operatorname{det} J_{\sigma}(\mathbf{x}) d \mathbf{x},
\end{aligned}
$$

for every $v \in H^{1}(\Omega)$. A radiation condition is implicitly incorporated in (2.4) by imposing that $w \in H^{1}(\Omega)$.

The equivalence of this complex scaling radiation condition to an expansion of the eigenfunctions in (spherical) Hankel functions of the first kind is shown in $[6,10,21]$ for resonances $\omega$ with $\Im(\sigma \omega)>0$ and scaling parameters $\sigma$ with positive real and imaginary part. The equivalence to a radiation condition based on boundary integrals (see [7]) is addressed in [23,32]. Note that a resonance $\omega$, as well as the interior part of the corresponding complex-scaled resonance function, is independent of $\sigma$ as long as $\Im(\sigma \omega)>0$.

An alternative approach to derive (2.4) which is also useful for dealing with inhomogeneous exterior domains is inspired by [14, Section 2.2]. We multiply (2.3) with specific test functions $\bar{g}$ of the form $\bar{g}(\mathbf{x}(\xi, \hat{\mathrm{x}}))=c(\hat{\mathrm{x}}) \exp (i k \xi)$ for $\mathbf{x} \in \Omega_{\text {ext }}$ with $\Im(k)>-\Im(\omega)$. Hence, the weak form

$$
\int_{\Omega_{\mathrm{ext}}} \nabla u(\mathbf{x}) \cdot \overline{\nabla g(\mathbf{x})} d \mathbf{x}=\omega^{2} \int_{\Omega_{\mathrm{ext}}} p(\mathbf{x}) u(\mathbf{x}) \overline{g(\mathbf{x})} d \mathbf{x}
$$

of the exterior part of (2.3) is well-defined and the integrands decay exponentially for $\xi \rightarrow \infty$. It can be shown that there exists a holomorphic extension of the integrands with respect to $\xi>0$. Using Cauchy's integral theorem and the fact that for suitable 
$\sigma$ the integrands in (2.5) decay exponentially along the path $\mathbf{x}(\sigma \xi, \hat{\mathrm{x}})$ for $\xi \rightarrow \infty$ and any $\hat{\mathrm{x}} \in \Gamma$, we may replace the original integration over $\Omega_{\mathrm{ext}}$ by the integration over the set $\gamma\left(\Omega_{\text {ext }}\right)$. An application of the transformation rule then leads to the weak formulation.

\subsection{The complex-scaled problem for inhomogeneous exterior domains}

Contrary to above we also consider potential functions $p$ which are not constant in $\Omega_{\text {ext }}$. In this case we state the problem under investigation in this paper as follows.

Problem 2.1 (complex-scaled resonance problem) Let $\Omega, \Omega_{\text {int }}, \Omega_{\text {ext }}, \Gamma$ be such that the assumptions in the beginning of Section 2.1 are fulfilled. Moreover let $\sigma \in \mathbb{C} \backslash\{0\}$ be a constant and $p_{\sigma} \in L^{\infty}(\Omega)$ be a complex valued function such that there holds

- in $\Omega_{\text {int }}: p_{\text {int }}:=\left.p_{\sigma}\right|_{\Omega_{\text {int }}}$ is real valued with $p_{\text {int }} \geq 1$, and

- in $\Omega_{\text {ext }} \cup \Gamma$ : for $\hat{\mathrm{x}} \in \Gamma$ and $\xi \geq 0$ there holds $p_{\sigma}(\mathbf{x}(\xi, \hat{\mathrm{x}}))=\hat{p}(\hat{\mathrm{x}}) \tilde{p}_{\sigma}(\xi)$, where $\hat{p}$ real valued with $\hat{p} \geq 1$, and $\tilde{p}_{\sigma}(\xi)$ is complex valued and converges to 1 for $\xi \rightarrow \infty$.

Then the resonance problem consists of finding resonances $\omega \in \mathbb{C}^{+}:=\{z \in \mathbb{C}$ : $\Im(\sigma \omega)>0\}$ with corresponding resonance functions $u \in H^{1}(\Omega) \backslash\{0\}$ such that

$$
s_{\text {int }}(u, v)+s_{\text {ext }}^{\sigma}(u, v)=\omega^{2}\left(m_{\text {int }}(u, v)+m_{\text {ext }}^{\sigma}(u, v)\right),
$$

for all $v \in H^{1}(\Omega)$. Thereby we define the interior sesquilinear forms by

$$
\begin{aligned}
m_{\mathrm{int}}(f, g) & :=\int_{\Omega_{\mathrm{int}}} p_{\mathrm{int}}(\mathbf{x}) f(\mathbf{x}) \overline{g(\mathbf{x})} d \mathbf{x}, \\
s_{\mathrm{int}}(f, g) & :=\int_{\Omega_{\mathrm{int}}} \nabla f(\mathbf{x}) \cdot \overline{\nabla g(\mathbf{x})} d \mathbf{x},
\end{aligned}
$$

and the exterior sesquilinear forms by

$$
\begin{aligned}
m_{\mathrm{ext}}^{\sigma}(f, g): & =\int_{\Omega_{\mathrm{ext}}} p_{\sigma}(\mathbf{x}) f(\mathbf{x}) \overline{g(\mathbf{x})} \operatorname{det} J_{\sigma}(\mathbf{x}) d \mathbf{x} \\
s_{\mathrm{ext}}^{\sigma}(f, g) & :=\int_{\Omega_{\mathrm{ext}}}\left(J_{\sigma}(\mathbf{x})^{-T} \nabla f(\mathbf{x})\right) \cdot\left(J_{\sigma}(\mathbf{x})^{-T} \overline{\nabla g(\mathbf{x})}\right) \operatorname{det} J_{\sigma}(\mathbf{x}) d \mathbf{x}
\end{aligned}
$$

In the numerical experiments, as well as in our convergence analysis, we allow several kinds of non-constant potential functions $p$. All of these have the form $p(\mathbf{x})=$ $\tilde{p}(\xi(\mathbf{x})) \hat{p}(\hat{\mathbf{x}}(\mathbf{x}))$ for $\mathbf{x} \in \Omega_{\text {ext }}$. In Section 6.2.1 we use $\tilde{p} \equiv 1$ and piecewise constant $\hat{p}$ with $\hat{p}(\hat{\mathrm{x}}) \geq 1$. In Section 6.2.2

$$
\tilde{p}(\xi)=1+\tilde{\epsilon} \frac{(1+\xi)^{2}}{1+(1+\xi)^{4}}
$$

with parameters $\tilde{\epsilon} \geq 0$ and continuous $\hat{p}$ with $\hat{p}(\hat{\mathrm{x}}) \geq q>0$ are used. Since the complex coordinate stretching $\xi \rightarrow \sigma \xi$ influences $\tilde{p}$ only, for these examples $p_{\sigma}$ in 
Problem 2.1 is given by $p_{\text {int }}=\left.p\right|_{\Omega_{\text {int }}}$ and $\tilde{p}_{\sigma}(\xi)=\tilde{p}(\sigma \xi)$. Clearly, $p_{\text {int }}$ and $\hat{p}$ are independent of $\sigma$. Note that the assumptions $p_{\text {int }} \geq 1, \hat{p} \geq 1$, and $\tilde{p}_{\sigma}$ to 1 for $\xi \rightarrow \infty$ in Problem 2.1, which are necessary for the convergence analysis, can be relaxed using positive constants instead of 1 . Similar to the case of a homogeneous exterior the radiation condition is implicitly included in Problem 2.1 in the assumption that the resonance functions of the complex-scaled problem are elements of $H^{1}(\Omega)$ (i.e., they decay fast enough for $\|\mathbf{x}\| \rightarrow \infty$ to be square integrable).

\subsection{A brief discussion of the complex scaling radiation condition for inhomogeneous exterior}

In the case where $p \not \equiv 1$ in $\Omega_{\text {ext }}$, justifying the use of the complex-scaled weak formulation and therefore the complex scaling radiation condition is more delicate than as it is sketched in Section 2.2 for the homogeneous case. In the following, we discuss the difficulties occurring in the described specific configurations.

In (2.5) three types of integrals over $\Gamma$ appear: The integral on the left-hand side splits into a standard $L^{2}(\Gamma)$ scalar product and the sesquilinear form induced by the negative Laplace-Beltrami operator. Additionally, we have a weighted $L^{2}(\Gamma)$-inner product with weight function $\hat{p}$ on the right-hand side.

Pure radial inhomogeneity: For $\Gamma=\partial B_{1}(0)$ and $p(\mathbf{x})=\tilde{p}(\xi(\mathbf{x}))$, i.e., $\hat{p} \equiv 1$, as in the homogeneous case, the spherical harmonics diagonalize all these three integrals. Along the lines of [16, 17], and in particular using a separation into Bessel-like equations, for rational potentials as used in Section 6.2.2 for some of the numerical experiments, a solution representation by functions similar to spherical Hankel functions of the first kind is possible. This allows the definition of a radiation condition and the application of similar arguments as in the homogeneous case. However, one has to take into account that the holomorphic extension of such rational $\tilde{p}$ might have singularities. Thus, the application of Cauchy's integral theorem in (2.5) is limited to domains given by scaling parameters $\sigma$ with sufficiently small argument.

Surface inhomogeneity: If the potential $p$ is not constant with respect to $\hat{\mathrm{x}} \in \Gamma$, in general the eigenfunctions of the Laplace-Beltrami operator on $\Gamma$ are not orthogonal with respect to the weighted $L^{2}(\Gamma)$ scalar product with the weight function $\hat{p}$. Hence, they cannot diagonalize all three terms. Therefore, to the best of our knowledge, even the definition of a radiation condition is not perfectly clear for such problems. Nevertheless, under reasonable conditions the convergence theory for Problem 2.1 holds true for surface inhomogeneities as well. Moreover using Cauchy's integral theorem as above one can argue that for suitable scaling parameters $\sigma$, the complex scaling radiation condition is in fact independent of $\sigma$.

\section{The discrete problem}

In this section we exploit the inherent structure of the exterior domain to derive a simple way of discretizing it without having to mesh it explicitly. 


\subsection{Abstract form of the discrete problem}

Our goal is to discretize Problem 2.1. To this end we pick $\mathcal{N} \in \mathbb{N}$ and a family of functions $\mathcal{B}_{\mathcal{N}}:=\left\{b_{0}, \ldots, b_{\mathcal{N}}\right\} \subset H^{1}(\Omega)$ and define the discrete space $\mathcal{X}_{\mathcal{N}}$ by

$$
\mathcal{X}_{\mathcal{N}}:=\operatorname{span}\left(\mathcal{B}_{\mathcal{N}}\right) \subset H^{1}(\Omega) \text {. }
$$

Defining the mass and stiffness matrices by

$$
\mathbf{M}:=\left(m_{i, j}\right)_{i, j=0, \ldots, \mathcal{N}}, \quad \mathbf{S}:=\left(s_{i, j}\right)_{i, j=0, \ldots, \mathcal{N}}
$$

with entries

$$
m_{i, j}=m_{\text {int }}\left(b_{j}, b_{i}\right)+m_{\text {ext }}^{\sigma}\left(b_{j}, b_{i}\right), \quad s_{i, j}=s_{\text {int }}\left(b_{j}, b_{i}\right)+s_{\text {ext }}^{\sigma}\left(b_{j}, b_{i}\right)
$$

respectively, we may formulate the discrete problem as follows.

Problem 3.1 Find $(\omega, \mathbf{u}) \in \mathbb{C}^{+} \times \mathbb{C}^{\mathcal{N}+1} \backslash\{0\}$, such that

$$
\mathbf{S u}=\omega^{2} \mathbf{M u},
$$

where the matrices $\mathbf{S}, \mathbf{M}$ are given by (3.1).

The discrete Problem 3.1 can be solved using standard eigenvalue solvers (see, e.g., [27]). In the following our task will be to find a suitable basis $\mathcal{B}_{\mathcal{N}}$. In the subsections up to Section 4.2 we focus on the exterior problem. The full discrete space $\mathcal{X}_{\mathcal{N}}$ will be defined in Section 4.3.

\subsection{Exterior variational formulation}

For the remainder of this section we assume that there exists a finite set of diffeomorphisms covering $\Gamma$ and let $\varphi: M \subset \mathbb{R}^{2} \rightarrow K \subset \Gamma$ be one of these mappings. For the case $\Gamma=\left\{\mathbf{x} \in \mathbb{R}^{3}:\|\mathbf{x}\|=R\right\}$, it is well-known that such a set exists.

Lemma 3.2 We can calculate the Jacobian of the coordinate transformation

$$
\Psi_{\varphi}: \begin{cases}\mathbb{R}_{\geq 0} \times M & \rightarrow \Omega_{\text {ext }} \cup \Gamma, \\ (\xi, \eta) & \mapsto\left(1+\frac{\xi}{R}\right) \varphi(\eta),\end{cases}
$$

its inverse, and its determinant by

$$
\begin{aligned}
D \Psi_{\varphi}(\xi, \eta) & =\left(\frac{1}{R} \varphi(\eta),\left(1+\frac{\xi}{R}\right) D \varphi(\eta)\right), \\
\left(D \Psi_{\varphi}(\xi, \eta)\right)^{-1} & =\left(\begin{array}{c}
\frac{1}{R} \varphi(\eta)^{T} \\
\frac{1}{1+\frac{\xi}{R}} D \varphi(\eta)^{\dagger}
\end{array}\right), \\
\left|\operatorname{det} D \Psi_{\varphi}(\xi, \eta)\right| & =\left(1+\frac{\xi}{R}\right)^{2} \sqrt{\left|\operatorname{det}\left(D \varphi(\eta)^{T} D \varphi(\eta)\right)\right|}
\end{aligned}
$$

where $A^{\dagger}:=\left(A^{T} A\right)^{-1} A^{T}$ is the pseudo inverse of a matrix $A \in \mathbb{C}^{3 \times 2}$ with full rank. 
Proof The Jacobian can be obtained by straightforward differentiation. Its inverse can be easily verified using the facts that $\varphi(\eta)^{T} D \varphi(\eta)=0, D \varphi(\eta)^{\dagger} D \varphi(\eta)=I$, and $\|\varphi(\eta)\|^{2}=R^{2}$.

For obtaining the determinant we calculate

$$
\begin{aligned}
\operatorname{det}\left(D \Psi_{\varphi}^{T} D \Psi_{\varphi}\right) & =\operatorname{det}\left(\left(\begin{array}{c}
\frac{1}{R} \varphi(\eta)^{T} \\
\left(1+\frac{\xi}{R}\right) D \varphi(\eta)^{T}
\end{array}\right)\left(\frac{1}{R} \varphi(\eta),\left(1+\frac{\xi}{R}\right) D \varphi(\eta)\right)\right) \\
& =\operatorname{det}\left(\begin{array}{l}
1 \\
0\left(1+\frac{\xi}{R}\right)^{2} D \varphi(\eta)^{T} D \varphi(\eta)
\end{array}\right) \\
& =\left(1+\frac{\xi}{R}\right)^{4} \operatorname{det}\left(D \varphi(\eta)^{T} D \varphi(\eta)\right) .
\end{aligned}
$$

By taking the square root we obtain the desired result.

Definition 3.3 Let $M \subset \mathbb{R}^{2}$ and $\varphi: M \rightarrow \Gamma$ be a diffeomorphism. Then we define the surface gradient on $\Gamma$ of a function $f: \Gamma \rightarrow \mathbb{C}$ by

$$
\hat{\nabla} f(\varphi(\eta)):=\left(D \varphi(\eta)^{\dagger}\right)^{T} \nabla_{\eta}(f \circ \varphi)(\eta) .
$$

It can be shown that the surface gradient $\hat{\nabla}$ defined above is independent of the specific embedding $\varphi$.

Theorem 3.4 Let $f, g \in H^{1}\left(\Omega_{\text {ext }}\right)$ and $\breve{f}(\xi, \hat{x}):=f(\mathbf{x}(\xi, \hat{x})), \breve{g}(\xi, \hat{x}):=g(\mathbf{x}(\xi, \hat{x}))$. Then the exterior sesquilinear forms from (2.7) can be rewritten in the coordinates $\xi, \hat{x}$ by

$$
\begin{aligned}
m_{\mathrm{ext}}^{\sigma}(f, g) & =\sigma \int_{\mathbb{R}_{>0} \times \Gamma} \tilde{p}_{\sigma}(\xi) \hat{p}(\hat{x}) \breve{f}(\xi, \hat{x}) \overline{\breve{g}(\xi, \hat{x})}\left(1+\frac{\sigma \xi}{R}\right)^{2} d(\xi, \hat{x}), \\
s_{\mathrm{ext}}^{\sigma}(f, g) & =\frac{1}{\sigma} \int_{\mathbb{R}_{>0} \times \Gamma} \frac{\partial \breve{f}}{\partial \xi}(\xi, \hat{x}) \overline{\frac{\partial \breve{g}}{\partial \xi}(\xi, \hat{x})}\left(1+\frac{\sigma \xi}{R}\right)^{2} d(\xi, \hat{x}), \\
& +\sigma \int_{\mathbb{R}_{>0} \times \Gamma} \hat{\nabla} \breve{f}(\xi, \hat{x}) \cdot \overline{\hat{\nabla} \breve{g}(\xi, \hat{x})} d(\xi, \hat{x}),
\end{aligned}
$$

where integration over $\Gamma$ of a function $h: \Gamma \rightarrow \mathbb{C}$ means integration by the surface measure, i.e.,

$$
\int_{\varphi(M)} h(\hat{x}) d \hat{x}:=\int_{M} h(\varphi(\eta)) \sqrt{\left|\operatorname{det}\left(D \varphi(\eta)^{T} D \varphi(\eta)\right)\right|} d \eta .
$$

Proof Using the determinant calculated in Lemma 3.2 and the fact that

$$
\gamma\left(\Psi_{\varphi}(\xi, \eta)\right)=\Psi_{\varphi}(\sigma \xi, \eta)
$$


where we use the symbol $\Psi_{\varphi}$ for the extension of said function to $\mathbb{C} \times M$, we obtain

$$
\begin{aligned}
D\left(\gamma \circ \Psi_{\varphi}\right)(\xi, \eta) & =D \Psi_{\varphi}(\sigma \xi, \eta)\left(\begin{array}{cc}
\sigma & 0 \\
0 & \mathbf{I}_{2}
\end{array}\right), \\
\left(D\left(\gamma \circ \Psi_{\varphi}\right)(\xi, \eta)\right)^{-1} & =\left(\begin{array}{cc}
\frac{1}{\sigma} & 0 \\
0 & \mathbf{I}_{2}
\end{array}\right)\left(D \Psi_{\varphi}(\sigma \xi, \eta)\right)^{-1}, \\
\operatorname{det} D\left(\gamma \circ \Psi_{\varphi}\right)(\xi, \eta) & =\sigma \operatorname{det} D \Psi_{\varphi}(\sigma \xi, \eta) .
\end{aligned}
$$

After applying the transformation rule we immediately obtain the formula for $m_{\mathrm{ext}}^{\sigma}$.

For the formula for $s_{\text {ext }}^{\sigma}$ we calculate

$$
\begin{aligned}
\nabla f\left(\Psi_{\varphi}(\xi, \eta)\right) & =\left(D\left(\gamma \circ \Psi_{\varphi}\right)(\xi, \eta)\right)^{-T} \nabla_{\xi, \eta}\left(f \circ \Psi_{\varphi}\right)(\xi, \eta) \\
& =\left(\frac{1}{\sigma R} \varphi(\eta), \frac{1}{1+\frac{\xi \sigma}{R}}\left(D \varphi(\eta)^{\dagger}\right)^{T}\right)\left(\begin{array}{c}
\frac{\partial f \circ \Psi_{\varphi}}{\partial \xi}(\xi, \eta) \\
\nabla_{\eta}\left(f \circ \Psi_{\varphi}\right)(\xi, \eta)
\end{array}\right) \\
& =\frac{1}{\sigma R} \varphi(\eta) \frac{\partial \breve{f}}{\partial \xi}(\xi, \varphi(\eta))+\frac{1}{1+\frac{\sigma \xi}{R}} \hat{\nabla} \breve{f}(\xi, \varphi(\eta)) .
\end{aligned}
$$

Plugging this into the integral and applying the transformation rule leads to the desired result.

\subsection{Tensor product discretization of the exterior problem}

Let

$$
\begin{aligned}
& \tilde{\mathcal{B}}_{N}:=\left\{\phi_{n}: n=0, \ldots, N\right\} \subset H^{1}\left(\mathbb{R}_{>0}\right), \\
& \hat{\mathcal{B}}_{M}:=\left\{b_{j}: j=0, \ldots, M\right\} \subset H^{1}(\Gamma)
\end{aligned}
$$

be families of linearly independent functions. Note that the number of radial unknowns $N \in \mathbb{N}$ has to be distinguished from the number of global unknowns $\mathcal{N} \in \mathbb{N}$ introduced in Section 3.1. Then we define discrete spaces on $\mathbb{R}_{>0}$ and $\Gamma$ respectively by

$$
\tilde{\mathcal{X}}_{N}:=\operatorname{span}\left(\tilde{\mathcal{B}}_{N}\right) \subset H^{1}\left(\mathbb{R}_{>0}\right)
$$

and

$$
\hat{\mathcal{X}}_{M}:=\operatorname{span}\left(\hat{\mathcal{B}}_{M}\right) \subset H^{1}(\Gamma) .
$$

To discretize the exterior problem, we use a tensor product space of the form

$$
\tilde{\mathcal{X}}_{N} \otimes \hat{\mathcal{X}}_{M}:=\operatorname{span}\left\{\phi \otimes b:(\xi, \hat{\mathrm{x}}) \mapsto \phi(\xi) b(\hat{\mathrm{x}}): \phi \in \tilde{\mathcal{B}}_{N}, b \in \hat{\mathcal{B}}_{M}\right\}
$$

To obtain the entries of the mass and stiffness matrices defined in (3.1) and (3.2), we need to evaluate the exterior sesquilinear forms for all pairs of basis functions. Since our basis functions are composed of a radial and a tangential part, we can decompose the sesquilinear forms accordingly and obtain for $\tilde{f}, \tilde{g} \in H^{1}\left(\mathbb{R}_{>0}\right)$ and $\hat{f}, \hat{g} \in H^{1}(\Gamma)$

$$
\begin{aligned}
& m_{\mathrm{ext}}^{\sigma}(\tilde{f} \otimes \hat{f}, \tilde{g} \otimes \hat{g})=\tilde{m}^{\sigma}(\tilde{f}, \tilde{g}) \hat{m}(\hat{f}, \hat{g}), \\
& s_{\mathrm{ext}}^{\sigma}(\tilde{f} \otimes \hat{f}, \tilde{g} \otimes \hat{g})=\tilde{s}_{1}^{\sigma}(\tilde{f}, \tilde{g}) \hat{s}_{0}(\hat{f}, \hat{g})+\tilde{s}_{0}^{\sigma}(\tilde{f}, \tilde{g}) \hat{s}_{1}(\hat{f}, \hat{g}),
\end{aligned}
$$


with

$$
\begin{aligned}
\tilde{m}^{\sigma}(\tilde{f}, \tilde{g}) & =\sigma \int_{0}^{\infty} \tilde{p}_{\sigma}(\xi)\left(1+\frac{\sigma \xi}{R}\right)^{2} \tilde{f}(\xi) \overline{\tilde{g}(\xi)} d \xi \\
\hat{m}(\hat{f}, \hat{g}) & =\int_{\Gamma} \hat{p}(\hat{\mathrm{x}}) \hat{f}(\hat{\mathrm{x}}) \overline{\hat{g}(\hat{\mathrm{x}})} d \hat{\mathrm{x}} \\
\tilde{s}_{1}^{\sigma}(\tilde{f}, \tilde{g}) & =\frac{1}{\sigma} \int_{0}^{\infty}\left(1+\frac{\sigma \xi}{R}\right)^{2} \tilde{f}^{\prime}(\xi) \overline{\tilde{g}^{\prime}(\xi)} d \xi \\
\hat{s}_{0}(\hat{f}, \hat{g}) & =\int_{\Gamma} \hat{f}(\hat{\mathrm{x}}) \overline{\hat{g}(\hat{\mathrm{x}})} d \hat{\mathrm{x}} \\
\tilde{s}_{0}^{\sigma}(\tilde{f}, \tilde{g}) & =\sigma \int_{0}^{\infty} \tilde{f}(\xi) \overline{\tilde{g}(\xi)} d \xi \\
\hat{s}_{1}(\hat{f}, \hat{g}) & =\int_{\Gamma} \hat{\nabla} \hat{f}(\hat{\mathrm{x}}) \cdot \overline{\hat{\nabla} \hat{g}(\hat{\mathrm{x}})} d \hat{\mathrm{x}} .
\end{aligned}
$$

A usual perfectly matched layer (PML) approach in this tensor product setting would be to truncate the set $\mathbb{R}_{>0}$ to some finite interval $(0, T)$ for $T>0$ and to use

$$
\tilde{\mathcal{X}}_{N} \subset H_{0}^{1}((0, T)):=\left\{f \in H^{1}\left(\mathbb{R}_{>0}\right): f(x)=0, x \geq T\right\},
$$

where $\tilde{\mathcal{X}}_{N}$ is a finite element space. Differing from this approach, we will choose basis functions with infinite support to omit truncation and ensure faster convergence. Our requirements to the basis functions $\phi_{n}$ and the discrete space $\tilde{\mathcal{X}}_{N}$ are:

(R 1) The basis functions $\phi_{n}$ can be evaluated in a numerically stable manner,

(R 2) the radial part of the solution can be well approximated by functions from $\tilde{\mathcal{X}}_{N}$

(R 3) it is easy to couple the interior to the exterior problem,

(R 4) the integrals $\int_{\mathbb{R}_{>0}} q(\xi) \phi_{n}(\xi) \phi_{j}(\xi) d \xi$ and $\int_{\mathbb{R}_{>0}} q(\xi) \phi_{n}^{\prime}(\xi) \phi_{j}^{\prime}(\xi) d \xi$ can be computed or approximated numerically for suitable coefficients $q$,

(R 5) the discretization matrices are sparse, and

(R 6) the condition numbers of the discretization matrices behave well for large values of $N$.

\section{Infinite elements based on complex scaling}

Complex scaling leads to solutions with anisotropic behavior. In the interior domain, as well as in the tangential direction of the exterior domain, the oscillating behavior of the function dominates. In radial direction of the exterior domain the exponential decay is crucial. Therefore, in order to reduce computational costs it is natural to choose suitable basis functions for the different parts of the solution.

Remark 4.1 Although we confined ourselves to an exterior domain which is (part of) the complement of a sphere, our method can be also applied to more general exterior domains $\Omega_{\text {ext }}$, if a parametrization of $\Omega_{\text {ext }}$ with respect to a surface and a radial 
coordinate and a decomposition of the sesquilinear forms into surface and radial parts similar to (3.3) exists (for details see [32, Section 7.1]).

In the case of cartesian scalings (see [22]) such a decomposition is not possible due to the existence of edge and corner regions. However, using tensor products of complex-scaled infinite elements in these edge and corner regions (as in [25, Section 2.3.1] for Hardy space infinite elements) makes our method applicable to cartesian scalings as well.

\subsection{Interior and interface discretization}

For discretizing the interior problem, basically any discrete space $\mathcal{X}_{\text {int }}=\operatorname{span}\left\{b_{j}\right.$ : $j=0, \ldots, L\} \subset H^{1}\left(\Omega_{\text {int }}\right)$ such that $\left.\mathcal{X}_{\text {int }}\right|_{\Gamma}:=\left\{\left.f\right|_{\Gamma}: f \in \mathcal{X}_{\text {int }}\right\} \subset H^{1}(\Gamma)$ can be used. The trace space of this interior discrete space is then used for the interface discretization (cf. Section 3), i.e.,

$$
\hat{\mathcal{X}}_{M}:=\left.\mathcal{X}_{\text {int }}\right|_{\Gamma}=\operatorname{span}\left\{\left.b_{j}\right|_{\Gamma}: j=0, \ldots, L\right\} \subset H^{1}(\Gamma) .
$$

In our examples we will choose $\mathcal{X}_{\text {int }}$ as a standard high-order conforming finite element space. Since in this case all of the basis functions corresponding to inner nodes in $\Omega_{\text {int }}$ are zero on the interface $\Gamma$, we expect the dimension of $\hat{\mathcal{X}}_{M}$ to be much smaller than the dimension of $\mathcal{X}_{\text {int }}$ in usual configurations.

\subsection{Radial discretization}

For the radial discretization we use the space of generalized Laguerre functions. These functions are used as basis functions of spectral methods for equations on unbounded domains with exponentially decreasing solutions (cf. [30, Section 7.4]). We will see in the following that they are a suitable choice considering our requirements (R 1)-(R 6). Following [30, Section 7.1], we define the generalized Laguerre polynomials and functions as follows.

Definition 4.2 For $n \in \mathbb{N}_{0}:=\{0,1, \ldots\}$ and $m \in \mathbb{Z}$, we define the generalized Laguerre polynomials by

$$
L_{n, m}(x):=\sum_{k=0}^{n}\left(\begin{array}{c}
n+m \\
n-k
\end{array}\right) \frac{(-x)^{k}}{k !},
$$

where the binomial coefficient is defined by $\left(\begin{array}{l}n \\ k\end{array}\right):=\prod_{j=1}^{k} \frac{n-j+1}{j}$ and we use the convention that an empty product equals one. Further we define the generalized Laguerre functions by

$$
\phi_{n, m}(x):=\exp (-x) L_{n, m}(2 x) .
$$

We shorten the notation by writing $\psi_{n}:=\phi_{n, 0}$ and $\phi_{n}:=\phi_{n,-1}$. Moreover, we define the radial discrete space by

$$
\tilde{\mathcal{X}}_{N}:=\operatorname{span}\left\{\phi_{n}: n=0, \ldots, N\right\} .
$$


Remark 4.3 (Comparison to Hardy space infinite elements) Hardy space infinite elements were first introduced in [14]. In Section 4.2 of [14], the Hardy space variational formulation is related to a complex-scaled variational formulation via a Fourier transform. If the isomorphism $\mathcal{Q}$ defined therein is applied to the Hardy space basis functions from [14, Section 2.4], we obtain the generalized Laguerre functions (see also [32, Section 6.4]). Hence, up to some minor modifications the discretization matrices of the complex-scaled infinite elements are the same as those of the Hardy space infinite element method in [14].

Note however that the functional framework of the complex-scaled infinite elements is more natural than the one of Hardy space infinite elements. The convergence analysis for Hardy space infinite elements in $[9,14]$ is very challenging and for resonance problems not complete. In the context of complex scalings on the other hand we are able to use standard approximation results of generalized Laguerre functions to derive a complete convergence analysis.

We proceed to study whether the basis functions defined in Definition 4.2 satisfy our requirements ( $\mathrm{R} 1)-(\mathrm{R} 6)$. To this end we state a few properties of the generalized Laguerre functions.

Lemma 4.4 (properties of the generalized Laguerre functions) Let $n \in \mathbb{N}_{0}, m \in \mathbb{Z}$ and $\phi_{n, m}, \phi_{n}, \psi_{n}$ as in Definition 4.2. Then

(i) for $j \in \mathbb{N}_{0}$,

$$
\int_{0}^{\infty} \xi^{m} \phi_{n, m}(\xi) \phi_{j, m}(\xi) d \xi=\frac{(n+m) !}{2^{m+1} n !} \delta_{n, j},
$$

and the functions $\psi_{n}, n \in \mathbb{N}_{0}$ form a complete orthogonal system of $L^{2}\left(\mathbb{R}_{>0}\right)$,

(ii) for $j, l \in \mathbb{N}_{0}$ such that $|j-n|>l+1$ and polynomials $p$ of degree $l$,

$$
\int_{0}^{\infty} p(\xi) \phi_{n}^{\prime}(\xi) \phi_{j}^{\prime}(\xi) d \xi=\int_{0}^{\infty} p(\xi) \phi_{n}(\xi) \phi_{j}(\xi) d \xi=0
$$

(iii)

$$
\phi_{n, m-1}=\phi_{n, m}-\phi_{n-1, m},
$$

$$
\phi_{n, m}^{\prime}=-\phi_{n, m}-2 \phi_{n-1, m+1}=-\phi_{n, m+1}-\phi_{n-1, m+1}=-\phi_{n, m}-2 \sum_{k=0}^{n-1} \phi_{k, m},
$$

$(v)$

$$
\phi_{n}(0)=\delta_{0, n},
$$

and

(vi) for $x \in \mathbb{C}$

$$
k \phi_{k, m}(x)=(2 k+m-1-2 x) \phi_{k-1, m}(x)-(k+m-1) \phi_{k-2, m}(x) .
$$


Proof All of the statements are easily checked by the reader and/or can be found, e.g., in [1, Chapter 22]

Item (v) of Lemma 4.4 shows that only the first radial basis function has to be coupled to an interior basis function, i.e., (R 3) is fulfilled. Moreover, items (i) and (ii) tell us that the resulting matrices will be sparse (cf. (R 5)) as long as the radial coefficients are polynomials with low degree.

Since the functions $\psi_{n}$ form an orthogonal basis with respect to the $L^{2}\left(\mathbb{R}_{>0}\right)$-inner product, and our basis functions $\phi_{n}$ are linear combinations of them (cf. 4.4(iii)) it is plausible that our discretization matrices are well-conditioned. We refer to [32, Section 9.1] for numerical experiments underlining this fact.

\subsection{Coupling the interior and the exterior problem}

Since we want to create a conforming discrete space for the whole problem, we need to couple our interior and exterior discrete spaces in a manner such that the resulting space is equivalent to a subspace of $H^{1}(\Omega)$. We achieve this by using

$$
\mathcal{Y}:=\left\{\left(u_{\text {int }}, u_{\text {ext }}\right): u_{\text {int }} \in \mathcal{X}_{\text {int }}, u_{\text {ext }} \in \tilde{\mathcal{X}}_{N} \otimes \hat{\mathcal{X}}_{M},\left.u_{\text {int }}\right|_{\Gamma}=u_{\text {ext }}(0, \cdot)\right\} .
$$

With an embedding defined by

$$
\iota: \begin{cases}\mathcal{Y} & \rightarrow H^{1}(\Omega), \\ \iota((u, v))(\mathbf{x}) & := \begin{cases}u(\mathbf{x}), & \mathbf{x} \in \Omega_{\mathrm{int}}, \\ v(\xi(\mathbf{x}), \hat{\mathrm{x}}(\mathbf{x})), & \mathbf{x} \in \Omega_{\mathrm{ext}},\end{cases} \end{cases}
$$

we define the discrete space $\mathcal{X}_{\mathcal{N}}$ of Section 3.1 as

$$
\mathcal{X}_{\mathcal{N}}:=\iota(\mathcal{Y}) \subset H^{1}(\Omega) .
$$

To obtain a basis of $\mathcal{Y}$ we have to couple the basis functions such that the resulting functions are continuous. This can be done by identifying an interior basis function $b_{j}$ with non-vanishing trace on $\Gamma$ with the exterior basis function $\left.\phi_{0} \otimes b_{j}\right|_{\Gamma}$. Note that, due to the tensor product structure of the exterior space, the parts of $\mathbf{S}$ and $\mathbf{M}$ that correspond to the exterior domain can be assembled by computing the radial and interface part separately and tensorizing them appropriately.

\subsection{Stable evaluation and numerical integration}

The generalized Laguerre functions can be evaluated numerically by using the stable three-term recursion given in Lemma 4.4(vi). We use Gauss rules for $(0, \infty)$ with weighting function $\exp (-\cdot)$ to obtain exact quadrature rules for the Laguerre functions (see [30, Chapter 7.1.2]). This enables us to also deal with inhomogeneous potentials in the exterior domain which is not possible in a straightforward way using classical Hardy space infinite elements. 


\section{Analysis}

The analysis of our method is based on the results of Karma [18, 19]. This work deals with (linear) Fredholm operators, which depend holomorphically on a complex parameter. The parameters for which the Fredholm operator is not uniquely invertible are the sought eigenvalues. For a certain class of approximations of these operators spectral convergence of the approximated eigenvalues is shown. In [10, Theorem 3.17] or [11] it is shown that these results are applicable for compact perturbations of coercive bilinear forms and their Galerkin discretizations.

Note that the standard eigenvalue convergence theory [2] of the Laplace operator cannot be used for the complex-scaled resonance problem (2.6) since for unbounded domains $\Omega$ the embedding $H^{1}(\Omega) \subset L^{2}(\Omega)$ is not compact. Hence, the solution operator to (2.6) is not compact, which would be the starting point of the theory in [2]. Therefore, rigorous convergence studies for complex-scaled resonance problems are very rare. In $[21,22]$ the error induced by the truncation to a perfectly matched layer is studied for specific scattering profiles. Subsequently, on the bounded, truncated domain standard arguments are used. [15] introduces the interpretation of a perfectly matched layer as a projection method for a waveguide structure. Based on this some basic convergence results are obtained. Finally, [10] or [12] give a full convergence analysis for a class of perfectly matched layer methods based on the results of $[18,19]$.

In this section we show that the complex-scaled infinite elements fit into this framework as well. Moreover, we derive convergence rates. Note that parts of the analysis are already contained in [32] although the analysis therein is not complete. However, in [32, Section 6.5.2] the approximation of spherical Hankel functions by Laguerre functions is studied. These studies can be helpful for choosing suitable scaling parameters.

\subsection{Weak coercivity}

We define the weak coercivity of an operator as follows.

Definition 5.1 For a Hilbert space $X$, let $A \in \mathcal{L}(X)$ (i.e., $A$ is a bounded, linear operator that maps $X$ to $X$ ). Then we call $A$ weakly coercive if the induced bilinear form is weakly coercive, i.e., there exists a compact operator $K \in \mathcal{L}(X)$ and a constant $C>0$ such that

$$
\left|((A+K) u, u)_{X}\right| \geq C\|u\|_{X}^{2},
$$

for all $u \in X$.

Next we show that the sesquilinear form induced by the eigenvalue problem in question is bounded for every fixed frequency $\omega$.

Lemma 5.2 Let $\sigma \in \mathbb{C} \backslash\{0\}$ and $\omega \in \mathbb{C}$. Then the sesquilinear form

$$
a_{\sigma}(\omega):=s_{\mathrm{int}}+s_{\mathrm{ext}}^{\sigma}-\omega^{2}\left(m_{\mathrm{int}}+m_{\mathrm{ext}}^{\sigma}\right)
$$


(with sesquilinear forms as defined in (2.7)) is bounded with respect to the $H^{1}(\Omega)$ norm.

Proof Using the surface gradient $\hat{\nabla}$ and the coordinates $(\xi(\mathbf{x}), \hat{\mathrm{x}}(\mathbf{x}))$ we can rewrite the $H^{1}\left(\Omega_{\text {ext }}\right)$-inner product for $f, g \in H^{1}\left(\Omega_{\text {ext }}\right)$ by

$$
\begin{aligned}
& \int_{\Omega_{\mathrm{ext}}} f(\mathbf{x}) \overline{g(\mathbf{x})}+\nabla f(\mathbf{x}) \cdot \overline{\nabla g(\mathbf{x})} d \mathbf{x}=\int_{\mathbb{R}_{>0} \times \Gamma}\left(1+\frac{\xi}{R}\right)^{2} \breve{f}(\xi, \hat{\mathrm{x}}) \overline{\breve{g}(\xi, \hat{\mathrm{x}})} d(\xi, \hat{\mathrm{x}}) \\
& +\int_{\mathbb{R}_{>0} \times \Gamma}\left(1+\frac{\xi}{R}\right)^{2} \frac{\partial}{\partial \xi} \breve{f}(\xi, \hat{\mathrm{x}}) \frac{\partial}{\partial \xi} \overline{\breve{g}(\xi, \hat{\mathrm{x}})} d(\xi, \hat{\mathrm{x}}) \\
& +\int_{\mathbb{R}_{>0} \times \Gamma} \hat{\nabla} \breve{f}(\xi, \hat{\mathrm{x}}) \cdot \hat{\nabla} \overline{\breve{g}(\xi, \hat{\mathrm{x}})},
\end{aligned}
$$

where we again used the notation $\breve{f}(\xi, \hat{\mathrm{x}}):=f(\mathbf{x}(\xi, \hat{\mathrm{x}})), \breve{g}(\xi, \hat{\mathrm{x}}):=g(\mathbf{x}(\xi, \hat{\mathrm{x}}))$. Then the boundedness follows from Theorem 3.4, the fact that $p_{\text {int }}$ and $p_{\sigma}$ are bounded, and straightforward computations.

Due to the previous Lemma 5.2 we may use the Riesz representation theorem to define the bounded operator $A_{\sigma}(\omega) \in \mathcal{L}\left(H^{1}(\Omega)\right)$ that satisfies

$$
\left(A_{\sigma}(\omega) f, g\right)_{H^{1}(\Omega)}=a_{\sigma}(\omega)(f, g),
$$

for every $\omega \in \mathbb{C}$ and $f, g \in H^{1}(\Omega)$. The following lemma is helpful for showing compactness of an operator.

Lemma 5.3 Let $\Omega \subset \mathbb{R}^{d}$ and $m \in L^{\infty}(\Omega)$ such that

$$
\lim _{R \rightarrow \infty}\|m\|_{L^{\infty}\left(\Omega \cap B_{R}^{c}\right)}=0 .
$$

Then the multiplication operator defined by

$$
M_{m}: \begin{cases}H^{1}(\Omega) & \rightarrow L^{2}(\Omega), \\ f & \mapsto m(\cdot) f(\cdot),\end{cases}
$$

is compact.

Proof The proof is given in [10, Lemma 4.3]. Using the indicator function $\chi_{\Omega \cap B_{R}}$ defined by $\chi_{\Omega \cap B_{R}}(\mathbf{x})=1$ for $\mathbf{x} \in \Omega \cap B_{R}$ and 0 elsewhere, the main tool of this proof is the operator $K_{R}: H^{1}(\Omega) \rightarrow L^{2}(\Omega)$ defined by $f \mapsto f \chi_{\Omega \cap B_{R}}$, which is compact due to the compact embedding $H^{1}\left(\Omega \cap B_{R}\right) \subset L^{2}\left(\Omega \cap B_{R}\right)$.

Using Lemma 5.3 we show that the operator $A_{\sigma}(\omega)$ is weakly coercive.

Lemma 5.4 For $\omega, \sigma \in \mathbb{C}$ such that $\Im(\omega) \leq 0$, $\Im(\sigma \omega)>0$ and $\arg (\sigma) \in\left[0, \frac{\pi}{2}\right)$ the operator $A_{\sigma}(\omega)$ given by (5.1) is weakly coercive. 
Proof We define the sesquilinear forms $b_{\sigma}(\omega), k_{\sigma}(\omega)$ by

$$
\begin{aligned}
b_{\sigma}(\omega)(f, g): & \int_{\Omega_{\mathrm{int}}} \nabla f(\mathbf{x}) \cdot \overline{\nabla g(\mathbf{x})} d \mathbf{x}+\int_{\Omega_{\mathrm{int}}} f(\mathbf{x}) \overline{g(\mathbf{x})} d \mathbf{x} \\
& +\frac{1}{\sigma} \int_{\mathbb{R}_{>0} \times \Gamma}\left(1+\frac{\sigma \xi}{R}\right)^{2} \frac{\partial}{\partial \xi} \breve{f}(\xi, \hat{\mathrm{x}}) \overline{\frac{\partial}{\partial \xi} \breve{g}(\xi, \hat{\mathrm{x}})} d(\xi, \hat{\mathrm{x}}) \\
& +\sigma \int_{\mathbb{R}_{>0} \times \Gamma} \hat{\nabla} \breve{f}(\xi, \hat{\mathrm{x}}) \cdot \overline{\hat{\nabla} \breve{g}(\xi, \hat{\mathrm{x}})} d(\xi, \hat{\mathrm{x}}) \\
& -\sigma^{3} \omega^{2} \int_{\mathbb{R}_{>0} \times \Gamma} \hat{p}(\hat{\mathrm{x}})\left(1+\frac{\xi}{R}\right)^{2} \breve{f}(\xi, \hat{\mathrm{x}}) \overline{\breve{g}(\xi, \hat{\mathrm{x}})} d(\xi, \hat{\mathrm{x}})
\end{aligned}
$$

and

$$
\begin{gathered}
k_{\sigma}(\omega)(f, g):=-\omega^{2} \int_{\Omega_{\mathrm{int}}} p_{\text {int }}(\mathbf{x}) f(\mathbf{x}) \overline{g(\mathbf{x})} d \mathbf{x}-\int_{\Omega_{\mathrm{int}}} f(\mathbf{x}) \overline{g(\mathbf{x})} d \mathbf{x} \\
-\sigma \omega^{2} \int_{\mathbb{R}_{>0} \times \Gamma} \hat{p}(\hat{\mathrm{x}})\left[\tilde{p}_{\sigma}(\xi)\left(1+\frac{\sigma \xi}{R}\right)^{2}-\sigma^{2}\left(1+\frac{\xi}{R}\right)^{2}\right] \breve{f}(\xi, \hat{\mathrm{x}}) \overline{\breve{g}(\xi, \hat{\mathrm{x}})} d(\xi, \hat{\mathrm{x}})
\end{gathered}
$$

for $f, g \in H^{1}(\Omega)$.

Clearly,

$$
a_{\sigma}(\omega)=b_{\sigma}(\omega)+k_{\sigma}(\omega) .
$$

The sesquilinear form $k_{\sigma}(\omega)$ can be rewritten as $k_{\sigma}(\omega)(f, g)=\left(f, M_{\bar{m}} g\right)_{L^{2}(\Omega)}$ with the function $m \in L^{\infty}(\Omega)$ given by $m(\mathbf{x})=-\omega^{2} p_{\text {int }}(\mathbf{x})-1$ for $\mathbf{x} \in \Omega_{\text {int }}$ and

$$
m(\mathbf{x}(\xi, \hat{\mathrm{x}}))=-\sigma \omega^{2} \hat{p}(\hat{\mathrm{x}}) \frac{\tilde{p}_{\sigma}(\xi)\left(1+\frac{\sigma \xi}{R}\right)^{2}-\sigma^{2}\left(1+\frac{\xi}{R}\right)^{2}}{\left(1+\frac{\xi}{R}\right)^{2}}
$$

for $\xi \in \mathbb{R}_{\geq 0}, \hat{\mathrm{x}} \in \Gamma \cdot M_{\bar{m}}: H^{1}(\Omega) \rightarrow L^{2}(\Omega)$ is the multiplication operator by $\bar{m}$, where we remind the reader that $\xi(\mathbf{x})=\|\mathbf{x}\|-R$ and $\lim _{\xi \rightarrow \infty} \tilde{p}_{\sigma}(\xi)=1$ (cf. Problem 2.1). Since

$$
\lim _{\xi \rightarrow \infty} m(\mathbf{x}(\xi, \hat{x}))=0
$$

uniformly in $\hat{\mathrm{x}} \in \Gamma$, we can apply Lemma 5.3 and obtain that $M_{\bar{m}}$ is compact. By [20, Theorem 4.10] the adjoint of a compact operator is also compact. Therefore, also

$$
K_{\sigma}(\omega):=\left.M_{\bar{m}}^{*}\right|_{H^{1}(\Omega)}: H^{1}(\Omega) \rightarrow H^{1}(\Omega),
$$

is compact. Clearly, $K_{\sigma}(\omega)$ is the operator induced by the sesquilinear form $k_{\sigma}(\omega)$.

It is easy to check that the sesquilinear form $b_{\sigma}(\omega)$ is bounded with respect to the $H^{1}(\Omega)$-norm. Thus, by means of the Riesz representation theorem we can define a bounded operator $B_{\sigma}(\omega)$ such that $\left(B_{\sigma}(\omega) f, g\right)_{H^{1}(\Omega)}:=b_{\sigma}(\omega)(f, g)$ for all $f, g \in$ $H^{1}(\Omega)$. 
Next, we show that $B_{\sigma}(\omega)$ is coercive. Since we assumed that $\arg (\sigma) \in\left[0, \frac{\pi}{2}\right)$, $\Im(\omega) \leq 0$, and $\Im(\sigma \omega)>0$, we obtain $\arg (\sigma \omega) \in\left(0, \frac{\pi}{2}\right)$, and therefore also $\Im\left(\sigma^{2} \omega^{2}\right)>0$. Because of this and $\Re\left(\frac{i}{\sigma^{2}}\right)>0$ we can find $0<\varepsilon<\frac{\pi}{2}$ such that

$$
\Re\left(\frac{i \exp (-i \varepsilon)}{\sigma^{2}}\right)>0 \text { and } \Im\left(\exp (-i \varepsilon) \sigma^{2} \omega^{2}\right)>0 \text {. }
$$

We use this to bound for $f \in H^{1}(\Omega)$ the four coefficients of the integrals below

$$
\begin{aligned}
\left|\frac{b_{\sigma}(\omega)(f, f)}{\sigma}\right| \geq & \Re\left(\frac{i \exp (-i \varepsilon)}{\sigma} b_{\sigma}(\omega)(f, f)\right) \\
& =\Re\left(\frac{i \exp (-i \varepsilon)}{\sigma}\right) \int_{\Omega_{\text {int }}}\|\nabla f(\mathbf{x})\|^{2}+|f(\mathbf{x})|^{2} d \mathbf{x} \\
& +\int_{\mathbb{R}_{>0} \times \Gamma}\left(\frac{i \exp (-i \varepsilon)\left(1+\frac{\sigma \xi}{R}\right)^{2}}{\sigma^{2}\left(1+\frac{\xi}{R}\right)^{2}}\right)\left(1+\frac{\xi}{R}\right)^{2}\left|\frac{\partial}{\partial \xi} \breve{f}(\xi, \hat{\mathrm{x}})\right|^{2} d(\xi, \hat{\mathrm{x}}) \\
& +\Re(i \exp (-i \varepsilon)) \int_{\mathbb{R}_{>0} \times \Gamma}\|\hat{\nabla} \breve{f}(\xi, \hat{\mathrm{x}})\|^{2} d(\xi, \hat{\mathrm{x}}) \\
& -\Re\left(i \exp (-i \varepsilon) \sigma^{2} \omega^{2}\right) \int_{\mathbb{R}_{>0} \times \Gamma} \hat{p}(\hat{\mathrm{x}})\left(1+\frac{\xi}{R}\right)^{2}|\breve{f}(\xi, \hat{\mathrm{x}})|^{2} d(\xi, \hat{\mathrm{x}}) .
\end{aligned}
$$

Firstly, we have

$$
\Re\left(\frac{i \exp (-i \varepsilon)}{\sigma}\right)=\frac{\sin (\varepsilon) \Re(\sigma)+\cos (\varepsilon) \Im(\sigma)}{|\sigma|^{2}}>0 .
$$

Secondly, because

$$
\arg \left(\frac{i \exp (-i \varepsilon)\left(1+\frac{\sigma \xi}{R}\right)^{2}}{\sigma^{2}\left(1+\frac{\xi}{R}\right)^{2}}\right)=\frac{\pi}{2}-\varepsilon+2 \arg \left(\frac{1}{\sigma}+\frac{\xi}{R}\right)
$$

converges monotonously towards $\frac{\pi}{2}-\varepsilon$ for $\xi \rightarrow \infty, \arg \left(\frac{i \exp (-i \varepsilon)}{\sigma^{2}}\right) \in\left(-\frac{\pi}{2}, \frac{\pi}{2}\right)$ by assumption and

$$
\left|\frac{1+\frac{\sigma \xi}{R}}{\sigma\left(1+\frac{\xi}{R}\right)}\right|=\left|\frac{\frac{1}{\sigma}+\frac{\xi}{R}}{1+\frac{\xi}{R}}\right|
$$

is bounded away from zero, we obtain that also $\Re\left(\frac{i \exp (-i \varepsilon)\left(1+\frac{\sigma \xi}{R}\right)^{2}}{\sigma^{2}\left(1+\frac{\xi}{R}\right)^{2}}\right)$ can be bounded away from zero uniformly in $\xi$.

For the third term we have $\mathfrak{R}(i \exp (-i \varepsilon))=\sin (\varepsilon)>0$. Lastly, we have

$$
-\mathfrak{R}\left(i \exp (-i \varepsilon) \sigma^{2} \omega^{2}\right)=\Im\left(\exp (-i \varepsilon) \sigma^{2} \omega^{2}\right)>0,
$$

and $\hat{p} \geq 1$ (cf. the assumptions in Problem 2.1). This gives that $b_{\sigma}(\omega)$, and therefore also $B_{\sigma}(\omega)$, is coercive. 
Lemma 5.4 shows that we may indeed apply the theory of Karma to the complex-scaled problem to obtain spectral convergence of our method. The approximation quality of an eigenvalue $\omega$ in a discrete space $\mathcal{X}_{\mathcal{N}}$ is governed by the best-approximation errors

$$
\inf _{u_{\mathcal{N}} \in \mathcal{X}_{\mathcal{N}}}\left\|u_{\mathcal{N}}-u\right\|_{H^{1}(\Omega)}, \quad \inf _{u_{\mathcal{N}} \in \mathcal{X}_{\mathcal{N}}}\left\|u_{\mathcal{N}}-u^{*}\right\|_{H^{1}(\Omega)},
$$

where $u$ are eigenfunctions and $u^{*}$ are eigenfunctions of the adjoint problem corresponding to the eigenvalue $\omega$ (cf. [10, Theorem 3.17]). Therefore, to prove convergence of our method we have to show that these errors tend to zero with respect to the discretization parameters.

Since the approximation error in the interior domain $\Omega_{\text {int }}$ can be treated using standard approximation results for $H^{1}$-conforming finite elements we focus on the approximation error in the exterior domain $\Omega_{\mathrm{ext}}$ in the following sections.

\subsection{Approximation of eigenfunctions by infinite elements}

Let $u \in H^{1}\left(\Omega_{\text {ext }}\right) \cap C^{\infty}\left(\Omega_{\text {ext }}\right)$ and, as before, $\breve{u}(\xi, \hat{\mathrm{x}}):=u(\mathbf{x}(\xi, \hat{\mathrm{x}}))$. For any fixed tangential variable $\hat{\mathrm{x}} \in \Gamma$ we can expand $\breve{u}(\cdot, \hat{\mathrm{x}})$ using Laguerre functions defined in 4.2

$$
\breve{u}(\xi, \hat{\mathrm{x}})=\sum_{n=0}^{\infty} \alpha_{n}(\hat{\mathrm{x}}) \psi_{n}(\xi)
$$

since the Laguerre functions $\psi_{n}$ form a complete orthogonal system in $L^{2}\left(\mathbb{R}_{>0}\right)$ (cf. Lemma 4.4(i)). The coefficients $\alpha_{n}(\hat{\mathrm{x}})$ are given by

$$
\alpha_{n}(\hat{\mathrm{x}})=2 \int_{0}^{\infty} \breve{u}(\xi, \hat{\mathrm{x}}) \psi_{n}(\xi) d \xi
$$

We additionally introduce the functions

$$
\begin{gathered}
u^{N}(\mathbf{x}(\xi, \hat{\mathrm{x}}))=\breve{u}^{N}(\xi, \hat{\mathrm{x}}):=\sum_{n=0}^{N} \alpha_{n}(\hat{\mathrm{x}}) \psi_{n}(\xi) \\
u^{N, h}(\mathbf{x}(\xi, \hat{\mathrm{x}}))=\breve{u}^{N, h}(\xi, \hat{\mathrm{x}}):=\sum_{n=0}^{N} \alpha_{n}^{h}(\hat{\mathrm{x}}) \psi_{n}(\xi)
\end{gathered}
$$

for fixed $N \in \mathbb{N}$ and the $L^{2}(\Gamma)$-orthogonal projections $\alpha_{n}^{h} \in \hat{\mathcal{X}}_{M}$ of $\alpha_{n}$ onto $\hat{\mathcal{X}}_{M}$.

To bound the best-approximation error of a function $u$ by discrete functions we proceed to derive a bound for the error

$$
\varepsilon(u, N, h):=\left\|u^{N, h}-u\right\|_{H^{1}\left(\Omega_{\mathrm{ext}}\right)} .
$$


To this end we decompose $\varepsilon(u, N, h)$ into a radial and a tangential part by

$$
\varepsilon(u, N, h) \leq \hat{\varepsilon}(u, N, h)+\tilde{\varepsilon}(u, N)
$$

with

$$
\begin{aligned}
& \hat{\varepsilon}(u, N, h):=\left\|u^{N, h}-u^{N}\right\|_{H^{1}\left(\Omega_{\mathrm{ext}}\right)}, \\
& \tilde{\varepsilon}(u, N):=\left\|u-u^{N}\right\|_{H^{1}\left(\Omega_{\mathrm{ext}}\right)} .
\end{aligned}
$$

In the following we analyze the dependence of these errors on the discretization parameters $N, h$.

\subsubsection{Super-algebraic decay of coefficients}

Proposition 5.5 Let $m, N \in \mathbb{N}, m \leq N+1$ and $f: \mathbb{R}_{>0} \rightarrow \mathbb{C}$ be such that $\xi \mapsto \xi^{k}\left(\frac{\partial}{\partial \xi}+\mathbf{I}\right)^{k} f(\xi) \in L^{2}\left(\mathbb{R}_{>0}\right)$ for all $k \in \mathbb{N}_{0}, k \leq m$. Then

$$
\left\|\Pi_{N} f-f\right\|_{L^{2}\left(\mathbb{R}_{>0}\right)}^{2} \leq \frac{(N-m+1) !}{(N+1) !}\left\|(\cdot)^{m}\left(\frac{\partial}{\partial \cdot}+\mathbf{I}\right)^{m} f\right\|_{L^{2}\left(\mathbb{R}_{>0}\right)}^{2}
$$

where $\Pi_{N}$ denotes the $L^{2}\left(\mathbb{R}_{>0}\right)$-orthogonal projection onto the space $\tilde{\mathcal{X}}_{N}$.

Proof [30, Theorem 7.9] with $\alpha=l=0$ and the function $f\left(\frac{\dot{2}}{2}\right)$. Note that the arguments of the basis functions in [30] are scaled by the factor $\frac{1}{2}$ compared to the basis functions $\psi_{j}$.

Proposition 5.5 shows that the interpolation error of smooth and sufficiently fast decaying functions on $\mathbb{R}_{>0}$ by (generalized) Laguerre functions decays superalgebraically. More precisely, if the right-hand side of (5.3) is bounded for each $m \in \mathbb{N}$, then the error on the left-hand side is bounded for each $m$ by the algebraic factor $C_{m, f} / N^{m}$ whereas $C_{m, f}$ depend on $m$ and $f$ but not on $N$. To obtain superalgebraic convergence of our method we need to assume such a behavior with respect to the radial direction of the complex-scaled resonance functions. For homogeneous exterior domains, this can, e.g., be proven by using boundary integral representations (cf. [31]).

Lemma 5.6 Let $u \in C^{\infty}\left(\overline{\Omega_{\text {ext }}}\right)$ and $\breve{u}(\xi, \hat{x}):=u(\mathbf{x}(\xi, \hat{x}))$ such that for every $\hat{x} \in \Gamma$ the function $\xi \mapsto \xi^{k}\left(\frac{\partial}{\partial \xi}+\mathbf{I}\right)^{k} \breve{u}(\xi, \hat{x})$ belongs to $L^{2}\left(\mathbb{R}_{>0}\right)$. Then for every $m \in \mathbb{N}$ there exists a constant $C>0$ such that

$$
\left\|\alpha_{n}\right\|_{L^{2}(\Gamma)} \leq C n^{-\frac{m}{2}}\left\|(\xi, \hat{x}) \mapsto \xi^{m}\left(\frac{\partial}{\partial \xi}+\mathbf{I}\right)^{m} \breve{u}(\xi, \hat{x})\right\|_{L^{2}\left(\mathbb{R}_{>0} \times \Gamma\right)},
$$

for all $n \in \mathbb{N}$, where $\alpha_{n}(\hat{x})=2 \int_{0}^{\infty} \breve{u}(\xi, \hat{x}) \psi_{n}(\xi) d \xi$. 
Proof For $\hat{\mathrm{x}} \in \Gamma$ we have by Proposition 5.5

$$
\begin{aligned}
\frac{1}{2} \sum_{n=N+1}^{\infty}\left|\alpha_{n}(\hat{\mathrm{x}})\right|^{2} & =\left\|\Pi_{N} \breve{u}(\cdot, \hat{\mathrm{x}})-\breve{u}(\cdot, \hat{\mathrm{x}})\right\|_{L^{2}\left(\mathbb{R}_{>0}\right)}^{2} \\
& \leq \frac{(N-m+1) !}{(N+1) !}\left\|(\cdot)^{m}\left(\frac{\partial}{\partial \cdot}+\mathbf{I}\right)^{m} \breve{u}(\cdot, \hat{\mathrm{x}})\right\|_{L^{2}\left(\mathbb{R}_{>0}\right)}^{2}
\end{aligned}
$$

Using Stirling's approximation for the factorial we obtain that there exists a constant $C>0$ such that

$$
\left|\alpha_{N}(\hat{\mathrm{x}})\right|^{2} \leq C N^{-m}\left\|(\cdot)^{m}\left(\frac{\partial}{\partial \cdot}+\mathbf{I}\right)^{m} \breve{u}(\cdot, \hat{\mathrm{x}})\right\|_{L^{2}\left(\mathbb{R}_{>0}\right)}^{2},
$$

for all $N \in \mathbb{N}$. By definition the functions $\hat{\mathrm{x}} \mapsto \alpha_{n}(\hat{\mathrm{x}})$ are continuous on $\Gamma$ by the bounded convergence theorem.

Similarly, the function $\hat{\mathrm{x}} \mapsto\left\|(\cdot)^{m}\left(\frac{\partial}{\partial \cdot}+\mathbf{I}\right)^{m} \breve{u}(\cdot, \hat{\mathrm{x}})\right\|_{L^{2}\left(\mathbb{R}_{>0}\right)}^{2}$ is continuous. Thus, we may integrate the right- and left-hand side of (5.4) over $\Gamma$ and obtain the claim.

\subsubsection{The tangential error}

The tangential error is given by

$$
\begin{aligned}
\hat{\varepsilon}(u, N, h)^{2} & =\left\|\sum_{n=0}^{N} \psi_{n}(\xi)\left(\alpha_{n}^{h}(\hat{\mathrm{x}})-\alpha_{n}(\hat{\mathrm{x}})\right)\right\|_{H^{1}\left(\Omega_{\mathrm{ext}}\right)}^{2} \\
& =\sum_{n, j=0}^{N}\left(\psi_{n} \hat{\varepsilon}_{n}, \psi_{j} \hat{\varepsilon}_{j}\right)_{H^{1}\left(\Omega_{\mathrm{ext}}\right)} \\
& =\sum_{n, j=0}^{N} \int_{0}^{\infty}\left(1+\frac{\xi}{R}\right)^{2} \psi_{n}(\xi) \psi_{j}(\xi) d \xi \int_{\Gamma} \hat{\varepsilon}_{n}(\hat{\mathrm{x}}) \overline{\hat{\varepsilon}_{j}(\hat{\mathrm{x}})} d \hat{\mathrm{x}} \\
& +\sum_{n, j=0}^{N} \int_{0}^{\infty}\left(1+\frac{\xi}{R}\right)^{2} \psi_{n}^{\prime}(\xi) \psi_{j}^{\prime}(\xi) d \xi \int_{\Gamma} \hat{\varepsilon}_{n}(\hat{\mathrm{x}}) \overline{\hat{\varepsilon}_{j}(\hat{\mathrm{x}})} d \hat{\mathrm{x}} \\
& +\sum_{n, j=0}^{N} \int_{0}^{\infty} \psi_{n}(\xi) \psi_{j}(\xi) d \xi \int_{\Gamma} \hat{\nabla} \hat{\varepsilon}_{n}(\hat{\mathrm{x}}) \hat{\nabla} \overline{\hat{\varepsilon}_{j}(\hat{\mathrm{x}})} d \hat{\mathrm{x}},
\end{aligned}
$$

where we used the notation

$$
\hat{\varepsilon}_{n}:=\alpha_{n}^{h}-\alpha_{n} .
$$

If we assume approximation properties of the tangential space of the form

$$
\begin{aligned}
\left\|\hat{\varepsilon}_{n}\right\|_{L^{2}(\Gamma)} & \leq \hat{C}_{0}(h)\left\|\alpha_{n}\right\|_{L^{2}(\Gamma)}, \\
\left\|\hat{\nabla} \hat{\varepsilon}_{n}\right\|_{L^{2}(\Gamma)} & \leq \hat{C}_{1}(h)\left\|\hat{\nabla} \alpha_{n}\right\|_{L^{2}(\Gamma)},
\end{aligned}
$$


for some functions $\hat{C}_{0}, \hat{C}_{1}$ with $\lim _{h \rightarrow 0} \hat{C}_{0}, \lim _{h \rightarrow 0} \hat{C}_{1}=0$, we obtain

$$
\begin{aligned}
\hat{\varepsilon}(u, N, h)^{2} & \leq \hat{C}_{0}(h)^{2} \sum_{n, j=0}^{N} \mathbf{M}_{n, j}^{\left(1+\frac{\xi}{R}\right)^{2}}\left\|\alpha_{n}\right\|_{L^{2}(\Gamma)}\left\|\alpha_{j}\right\|_{L^{2}(\Gamma)} \\
& +\hat{C}_{0}(h)^{2} \sum_{n=0}^{N}\left\|\hat{\nabla} \alpha_{n}\right\|_{L^{2}(\Gamma)}^{2} \\
& +\hat{C}_{1}(h)^{2} \sum_{n, j=0}^{N} \mathbf{S}_{n, j}^{\left(1+\frac{\xi}{R}\right)^{2}}\left\|\alpha_{n}\right\|_{L^{2}(\Gamma)}\left\|\alpha_{j}\right\|_{L^{2}(\Gamma)},
\end{aligned}
$$

where we use the notation

$$
\begin{aligned}
\mathbf{M}_{n, j}^{f} & :=\int_{0}^{\infty} f(\xi) \psi_{n}(\xi) \psi_{j}(\xi) d \xi \\
\mathbf{S}_{n, j}^{f} & :=\int_{0}^{\infty} f(\xi) \psi_{n}^{\prime}(\xi) \psi_{j}^{\prime}(\xi) d \xi
\end{aligned}
$$

for suitable functions $f$ such that the integrals are finite.

Remark 5.7 For the surface discretization, high-order $H^{1}$-conforming surface finite elements can be used. For the construction of these elements we refer to [8]. Such elements lead to error bounds $\hat{C}_{0}(h)=h^{k}, \hat{C}_{1}(h)=h^{k-1}$, where $k \in \mathbb{N}$ is the element order.

The following Lemma shows that the entries of the (infinite) matrices defined above grow at most polynomially.

Lemma 5.8 Let $n, j \in \mathbb{N}_{0}$ and $\mathbf{M}_{n, j}^{\left(1+\frac{\xi}{R}\right)^{2}}$ and $\mathbf{S}_{n, j}^{\left(1+\frac{\xi}{R}\right)^{2}}$ be as above. Then there exists a constant $C>0$ independent of $n, j$ such that

$$
\left|\mathbf{M}_{n, j}^{\left(1+\frac{\xi}{R}\right)^{2}}\right|,\left|\mathbf{S}_{n, j}^{\left(1+\frac{\xi}{R}\right)^{2}}\right| \leq C \min \left\{1, n^{2}, j^{2}\right\}
$$

Proof We exemplarily only prove the assertion for $\mathbf{S}_{n, j}^{\left(1+\frac{\xi}{R}\right)^{2}}$. For $n, j \in \mathbb{N}_{0}$ we decompose $\mathbf{S}_{n, j}^{\left(1+\frac{\xi}{R}\right)^{2}}$ into

$\mathbf{S}_{n, j}^{\left(1+\frac{\xi}{R}\right)^{2}}=\int_{0}^{\infty} \psi_{n}^{\prime}(\xi) \psi_{j}^{\prime}(\xi) d \xi+2 \int_{0}^{\infty} \frac{\xi}{R} \psi_{n}^{\prime}(\xi) \psi_{j}^{\prime}(\xi) d \xi+\int_{0}^{\infty} \frac{\xi^{2}}{R^{2}} \psi_{n}^{\prime}(\xi) \psi_{j}^{\prime}(\xi) d \xi$

Using the formula

$$
\psi_{n}^{\prime}=-\psi_{n}-2 \sum_{j=0}^{n-1} \psi_{j}
$$


from Lemma 4.4(iv), we obtain

$$
\begin{aligned}
\int_{0}^{\infty} \psi_{n}^{\prime}(\xi) \psi_{j}^{\prime}(\xi) d \xi & =\int_{0}^{\infty}\left(\psi_{n}(\xi)+2 \sum_{k=0}^{n-1} \psi_{k}(\xi)\right)\left(\psi_{j}(\xi)+2 \sum_{l=0}^{j-1} \psi_{l}(\xi)\right) d \xi \\
& = \begin{cases}\frac{1}{2}(1+4 n), & n=j, \\
\frac{1}{2}(2+4 \min \{n, j\}), & n \neq j .\end{cases}
\end{aligned}
$$

Using

$$
\int_{0}^{\infty}(2 \xi)^{k} \phi_{n, k}(\xi) \phi_{j, k}(\xi) d \xi=\frac{(n+k) !}{2 n !} \delta_{n, j}
$$

and

$$
\psi_{n}^{\prime}=-\phi_{n, 1}-\phi_{n-1,1}
$$

we obtain

$$
\begin{aligned}
2 \int_{0}^{\infty} \xi \psi_{n}^{\prime}(\xi) \psi_{j}^{\prime}(\xi) d \xi & =2 \int_{0}^{\infty} \xi\left(\phi_{n, 1}(\xi)+\phi_{n-1,1}(\xi)\right)\left(\phi_{j, 1}(\xi)+\phi_{j-1,1}(\xi)\right) d \xi \\
& = \begin{cases}\frac{1}{2}(2 n+1), & n=j, \\
\frac{1}{2}(n+1), & n+1=j, \\
\frac{1}{2} n, & n-1=j, \\
0, & \text { else. }\end{cases}
\end{aligned}
$$

By performing similar calculations for the quadratic term and the expression $\mathbf{M}_{n, j}^{\left(1+\frac{\xi}{R}\right)^{2}}$, we obtain the claim.

Using the previous Lemma and the fact that the $L^{2}(\Gamma)$-norm of the functions $\alpha_{n}, \hat{\nabla} \alpha_{n}$ decays super-algebraically in $n$ we can prove the following theorem:

Theorem 5.9 Let $u$ be an eigenfunction, $N \in \mathbb{N}$ and $h$ a surface discretization parameter such that (5.5) holds. Then the tangential error $\hat{\varepsilon}(u, N, h)$ can be bounded by

$$
\hat{\varepsilon}(u, N, h) \leq D \max \left\{\hat{C}_{0}(h), \hat{C}_{1}(h)\right\}
$$

for some constant $D>0$ depending on $u$ and independent of $N$ and $h$.

Proof Since $u$ is smooth we may apply Lemma 5.6 to obtain that the quantities $\left\|\alpha_{n}\right\|_{L^{2}(\Gamma)}$ and $\left\|\hat{\nabla} \alpha_{n}\right\|_{L^{2}(\Gamma)}$ decay super-algebraically with respect to the index $n$, i.e., for a given integer $m$ there exists a constant $C>0$ such that

$$
\left\|\alpha_{n}\right\|_{L^{2}(\Gamma)},\left\|\hat{\nabla} \alpha_{n}\right\|_{L^{2}(\Gamma)} \leq C n^{-\frac{m}{2}}
$$


Picking $m$ large enough we obtain

$\sum_{n, j=0}^{N}\left|\mathbf{M}_{n, j}^{\left(1+\frac{\xi}{R}\right)^{2}}\right|\left\|\alpha_{n}\right\|_{L^{2}(\Gamma)}\left\|\alpha_{j}\right\|_{L^{2}(\Gamma)} \leq \sum_{n, j=0}^{\infty}\left|\mathbf{M}_{n, j}^{\left(1+\frac{\xi}{R}\right)^{2}}\right|\left\|\alpha_{n}\right\|_{L^{2}(\Gamma)}\left\|\alpha_{j}\right\|_{L^{2}(\Gamma)} \leq D$

for a constant $D$ independent of $N$.

The same arguments can be repeated for the term containing $\mathbf{S}^{\left(1+\frac{\xi}{R}\right)^{2}}$. Using (5.5) we obtain the claim.

\subsection{The radial error}

The radial error is given by

$$
\begin{aligned}
\tilde{\varepsilon}(u, N)^{2} & =\left\|\sum_{n=N+1}^{\infty} \psi_{n}(\xi) \alpha_{n}(\hat{\mathrm{x}})\right\|_{H^{1}\left(\Omega_{\mathrm{ext}}\right)}^{2} \\
& =\sum_{n, j=N+1}^{\infty} \int_{0}^{\infty}\left(1+\frac{\xi}{R}\right)^{2} \psi_{n}(\xi) \psi_{j}(\xi) d \xi \int_{\Gamma} \hat{\alpha}_{n}(\hat{\mathrm{x}}) \overline{\hat{\alpha}_{j}(\hat{\mathrm{x}})} d \hat{\mathrm{x}} \\
& +\sum_{n, j=N+1}^{\infty} \int_{0}^{\infty}\left(1+\frac{\xi}{R}\right)^{2} \psi_{n}^{\prime}(\xi) \psi_{j}^{\prime}(\xi) d \xi \int_{\Gamma} \hat{\alpha}_{n}(\hat{\mathrm{x}}) \overline{\hat{\alpha}_{j}(\hat{\mathrm{x}})} d \hat{\mathrm{x}} \\
& +\sum_{n, j=N+1}^{\infty} \int_{0}^{\infty} \psi_{n}(\xi) \psi_{j}(\xi) d \xi \int_{\Gamma} \hat{\nabla} \hat{\alpha}_{n}(\hat{\mathrm{x}}) \hat{\nabla} \overline{\hat{\alpha}_{j}(\hat{\mathrm{x}})} d \hat{\mathrm{x}} .
\end{aligned}
$$

Using the same notation as above we obtain

$$
\begin{aligned}
\tilde{\varepsilon}(u, N)^{2} & \leq \sum_{n, j=N+1}^{\infty} \mathbf{M}_{n, j}^{\left(1+\frac{\xi}{R}\right)^{2}}\left\|\alpha_{n}\right\|_{L^{2}(\Gamma)}\left\|\alpha_{j}\right\|_{L^{2}(\Gamma)}+\delta_{n, j}\left\|\hat{\nabla} \alpha_{n}\right\|_{L^{2}(\Gamma)}\left\|\hat{\nabla} \alpha_{j}\right\|_{L^{2}(\Gamma)} \\
& +\sum_{n, j=N+1}^{\infty} \mathbf{S}_{n, j}^{\left(1+\frac{\xi}{R}\right)^{2}}\left\|\alpha_{n}\right\|_{L^{2}(\Gamma)}\left\|\alpha_{j}\right\|_{L^{2}(\Gamma)} .
\end{aligned}
$$

Using again the decay of the coefficients $\alpha_{n}$ we can prove the following theorem.

Theorem 5.10 Let $\tilde{\varepsilon}$ be as above, and $N \in \mathbb{N}$. Then for any $k \in \mathbb{N}$ there exists a constant $\tilde{C}$ depending only on $k$ and $u$ such that

$$
\tilde{\varepsilon}(u, N) \leq \tilde{C} N^{-k}
$$


Proof For any $m \in \mathbb{N}$ and $\mathbf{A}_{n, j}=\mathbf{M}_{n, j}^{\left(1+\frac{\xi}{R}\right)^{2}}, \mathbf{S}_{n, j}^{\left(1+\frac{\xi}{R}\right)^{2}}$ and $c_{j} \in \mathbb{C}, j \in \mathbb{N}_{0}$ s.t. $\left|c_{j}\right| \leq E j^{-\frac{m}{2}}$ for some $E>0$ we obtain by using Lemma 5.8

$$
\begin{aligned}
\sum_{n=N+1}^{\infty} c_{n} \sum_{j=N+1}^{\infty} \mathbf{A}_{n, j} \overline{c_{j}} \mid & \leq \sum_{n=N+1}^{\infty}\left|c_{n}\right| \sum_{j=N+1}^{\infty}\left|\mathbf{A}_{n, j} \overline{c_{j}}\right| \\
& \leq C E^{2} \sum_{n=N+1}^{\infty} n^{-\frac{m}{2}} \sum_{j=N+1}^{\infty} j^{2} j^{-\frac{m}{2}} \\
& \leq C E^{2} \sum_{n=N+1}^{\infty} n^{-\frac{m}{2}} \int_{N}^{\infty} x^{2-\frac{m}{2}} d x \\
& =C E^{2} \sum_{n=N+1}^{\infty} n^{-\frac{m}{2}} \frac{1}{\frac{m}{2}-3} N^{3-\frac{m}{2}} \\
& \leq C E^{2} \frac{1}{\left(\frac{m}{2}-3\right)\left(\frac{m}{2}-1\right)} N^{4-m}
\end{aligned}
$$

Replacing $c_{j}$ by $\left\|\alpha_{j}\right\|_{L^{2}(\Gamma)}$ and $\left\|\hat{\nabla} \alpha_{j}\right\|_{L^{2}(\Gamma)}$, summing over the expressions and choosing $m$ sufficiently large yields the claim.

\subsection{Summary of the convergence results}

In this subsection we sum up the main convergence results. First, we collect the assumptions made in the previous sections: We assume a linear complex scaling with parameter $\sigma \in \mathbb{C} \backslash\{0\}$ and an interface $\Gamma \subset \partial B_{R}$ with $R>0$. Moreover, we assume that

(C 1) $\omega$ is an eigenvalue of Problem 2.1 such that $\Im(\sigma \omega)>0$,

(C 2) $\left(N_{n}, h_{n}\right)$ is a sequence of discretization parameters with $\lim _{n \rightarrow \infty}\left(N_{n}, h_{n}\right)=$ $(\infty, 0)$,

(C 3) $u$ is an eigenfunction corresponding to $\omega$ and $u^{*}$ is an eigenfunction of the adjoint problem corresponding to $\bar{\omega}$ such that

(C 3a) $u, u^{*}$ and all their derivatives with respect to the radial variable decay super-algebraically, uniformly in the surface variable,

(C 3b) the surface discretizations are chosen such that (5.5) holds, and

(C 4) $\mathfrak{R}(\sigma), \Im(\sigma)>0$, and $\Im(\omega) \leq 0$.

Remark 5.11 (C 1) is mainly needed to ensure that the complex-scaled resonance functions decay exponentially in radial direction and to ensure that the resonances are independent of $\sigma$ (see for homogeneous exterior domains, e.g., [32]). (C 2) implies, together with the other assumptions, pointwise $H^{1}$-convergence of the projection 
operators onto the discrete spaces. The assumption (C 3) is needed to derive convergence rates. In particular, (C 3a) is used in Prop. 5.5 for the radial error and (C 3b) for the surface error. Finally, $(\mathrm{C} 4)$ is used to prove weak coercivity in Lem. 5.4.

The following Theorem 5.12 is basically a restatement of [10, Thm. 3.17.(i-iv)] for our specific case. It ensures that for each resonance of the continuous problem there exist sequences of discrete eigenvalues converging with the correct multiplicity towards this resonance. Moreover, there is no fail convergence, i.e., if a sequence of discrete eigenvalues converges, then the limit is a resonance of the continuous problem.

Theorem 5.12 Let $\sigma \in \mathbb{C} \backslash\{0\}$ and $R>0$ and let $(C 1-4)$ hold. Then

(i) there exists a sequence $\omega_{n}$ such that $\omega_{n} \rightarrow \omega$ with $\omega_{n}$ being an eigenvalue of the discrete problem corresponding to the discretization parameters $N_{n}, h_{n}$,

(ii) for any sequence of eigenpairs $\left(\omega_{n}, u_{n}\right)$ of the corresponding discrete problems with $\omega_{n} \rightarrow \omega_{0} \in\{\lambda \in \mathbb{C}: \Im(\lambda) \leq 0, \Im(\sigma \lambda)>0\}$ it follows that $\omega_{0}$ is an eigenvalue of Problem 2.1 and the cluster points of the sequence $u_{n}$ are eigenfunctions corresponding to $\omega_{0}$,

(iii) for every compact subset $\Lambda$ of the resolvent set of the continuous problem there exists an index $n_{0}$ such that $\Lambda$ contains no eigenvalues of the discrete problems corresponding to indices $n>n_{0}$, and

(iv) for every compact set $\Lambda \subset\{\lambda \in \mathbb{C}: \Im(\lambda) \leq 0$, $\Im(\sigma \lambda)>0\}$ such that its boundary is contained in the resolvent set of the continuous problem there exists an index $n_{0}$ such that the sums of the algebraic multiplicities of the eigenvalues in $\Lambda$ of the continuous and the discrete problems coincide for every $n>n_{0}$.

The following theorem is based on [10, Thm. 3.17.(v-vii)] and gives convergence rates. For simplicity we only state the result for eigenvalues with algebraic multiplicity one.

Theorem 5.13 Let $\sigma \in \mathbb{C} \backslash\{0\}, R>0$, and $\omega$ be an eigenvalue of Problem 2.1 such that $(C 1-4)$ hold. Then there exists a constant $c>0$ and an index $n_{0}$ such that

$$
\begin{aligned}
\left|\omega-\omega_{n}\right| & \leq c\left(\tilde{\varepsilon}\left(u, N_{n}\right)+\hat{\varepsilon}\left(u, N_{n}, h_{n}\right)\right)\left(\tilde{\varepsilon}\left(u^{*}, N_{n}\right)+\hat{\varepsilon}\left(u^{*}, N_{n}, h_{n}\right)\right), \\
\left\|u-u_{n}\right\|_{H^{1}(\Omega)} & \leq c\left(\left|\omega-\omega_{n}\right|+\tilde{\varepsilon}\left(u, N_{n}\right)+\hat{\varepsilon}\left(u, N_{n}, h_{n}\right)\right),
\end{aligned}
$$

for all $n>n_{0}$, where $\left(\omega_{n}, u_{n}\right)$ is a sequence of discrete eigenpairs with $\omega_{n} \rightarrow \omega$, and the approximation errors $\hat{\varepsilon}, \tilde{\varepsilon}$ are given by (5.2). For any $m \in \mathbb{N}$ and $f=u, u^{*}$ the errors can be bounded by

$$
\begin{aligned}
\tilde{\varepsilon}\left(f, N_{n}\right) & \leq \tilde{C} N_{n}^{-\frac{m}{2}}, \\
\hat{\varepsilon}\left(f, N_{n}, h_{n}\right) & \leq D \max \left\{\hat{C}_{0}\left(h_{n}\right), \hat{C}_{1}\left(h_{n}\right)\right\},
\end{aligned}
$$

for constants $\tilde{C}, D>0$ independent of $N_{n}, h_{n}$ and functions $\hat{C}_{0}, \hat{C}_{1}$ that satisfy (5.5) (cf. Remark 5.7). 


\section{Numerical experiments}

In the following we illustrate our theoretical findings from the previous sections by numerical examples. All numerical examples were computed using the high-order finite element software NGSolve [29] and the mesh generator Netgen [28].

\subsection{Comparison to perfectly matched layers}

In this subsection we compare the computational costs of our infinite elements and a conventional PML by approximating the resonances of Problem 2.1 on

$$
\Omega_{\mathrm{ext}}:=\Omega:=\mathbb{R}^{3} \backslash B_{1}(0)=\left\{\mathbf{x} \in \mathbb{R}^{3}:\|\mathbf{x}\|>1\right\},
$$

and $p \equiv 1$. In this situation, the resonances are the roots of the derivatives of spherical Hankel functions and can therefore be computed by solving a scalar equation.

All computations in this section were done on a desktop computer with an Intel i3 CPU with $2 \times 3.5 \mathrm{GHz}$ and $16 \mathrm{GiB}$ memory. The eigenvalues were calculated using a shift-and-invert Arnoldi algorithm (cf. [27]) and a direct inverse via a Cholesky factorization for complex symmetric matrices. All given times are for the factorization of the given system matrix only, since this is the main contribution to the overall computational costs. The largest problems in the examples had 99425 degrees of freedom (24 in radial direction). Factorizing the inverse took up about $7 \mathrm{GiB}$ of memory.

Figure 2 shows the error plotted against factorization times for infinite elements and a PML using the same tensor product method described in Section 3 but with one-dimensional high-order finite element basis functions in radial direction on an interval $(0, T)$. We applied uniform $h$-refinement to obtain a succession of discretizations. In Fig. $2 \mathrm{a}$ and $\mathrm{c}$ the error generated by the truncation of the exterior domain can be observed at approximately $10^{-3}$. In Fig. $2 \mathrm{c}$ and $\mathrm{d}$ the infinite elements already reach the error generated by the surface discretization which is approximately $10^{-7}$. All experiments show that the infinite elements are clearly superior to the used PML discretizations with respect to computational efficiency.

Note that due to the fact that we used the tensor product ansatz also for the PML discretizations, this version of PML is already more efficient than a typical PML based on an unstructured exterior mesh. On the other hand, there are many ways to optimize the PML even more, e.g., using non-uniform meshes on $(0, T)$ or nonlinear complex scalings. We are not claiming the complex-scaled infinite elements to be superior to all possible versions of perfectly matched layers. Nevertheless, for complex-scaled infinite elements only the scaling parameter $\sigma$ and the number of degrees of freedom in radial direction have to be chosen. Typically, for an efficient PML more parameters have to be optimized.

\subsection{Application of infinite elements to problems with inhomogeneous exterior domains}

As described in Section 2.4 the theoretical justification of complex scaling and the induced radiation condition for problems with an inhomogeneous exterior is more 

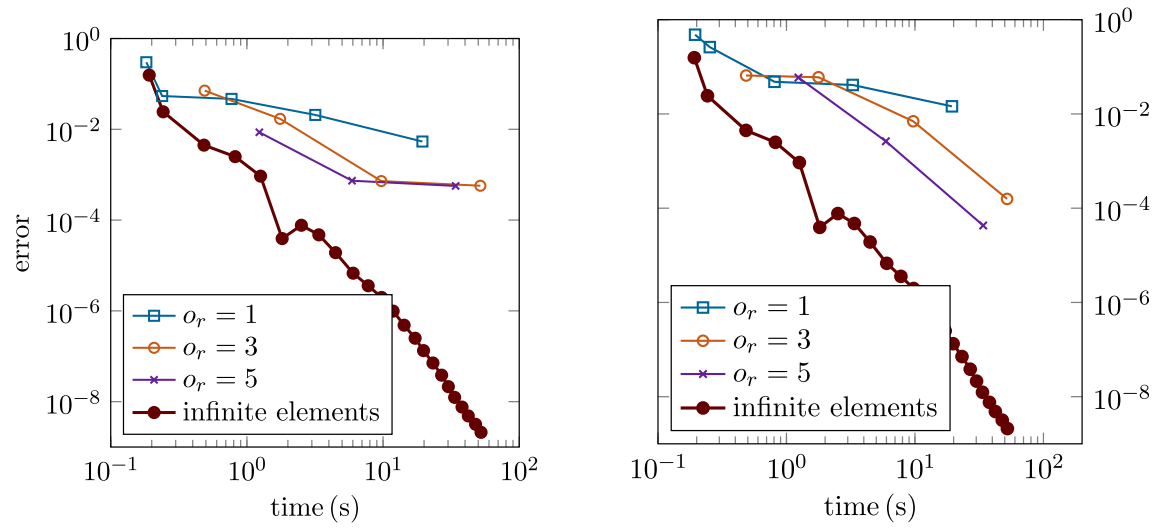

(a) $T=5$,

$\omega \approx 2.90391653245-1.20186645975 i$

(b) $T=8$,

$\omega \approx 2.90391653245-1.20186645975 i$
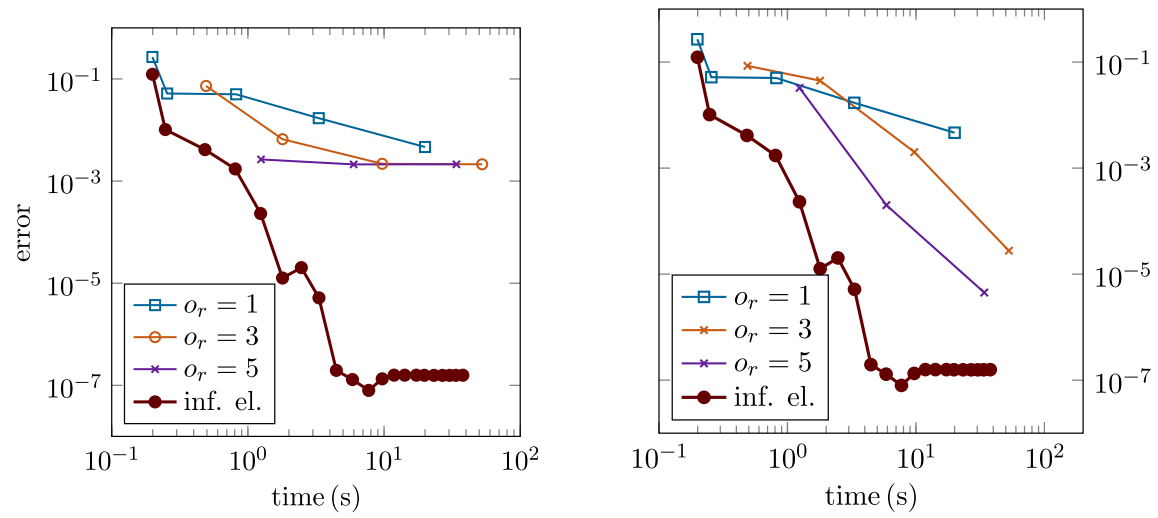

(c) $T=5$,

$\omega \approx 5.77658328455-1.41788771722 i$

(d) $T=8$,

$\omega \approx 5.77658328455-1.41788771722 i$

Fig. 2 Comparison of errors against factorization times for eigenvalues $\omega$ and a surface discretization with order $o=5$ and mesh size $h=0.3, \sigma=\frac{1+i}{\omega}$ and different exterior discretizations. We use PMLs with truncation at $T$ and radial elements of order $o_{r}$

delicate than in the homogeneous case. Therefore, in the following examples we start from well-justified reference configurations. We monitor the changes in the numerical results when using continuous perturbations of this configuration. In this way we make sure to obtain reasonable results.

\subsubsection{An example with a surface inhomogeneity}

We use an example with $\Omega=\mathbb{R}^{3}$ and a potential function $p$, which is piecewise constant. It takes three different values in one bounded (a sphere) and two unbounded (part of a cone and the remainder of $\mathbb{R}^{3}$ ) subdomains (cf. Fig. 3). In the cases $\alpha \in$ $\{0,2 \pi\}$ the exterior domain is homogeneous. Moreover, in these cases the problem 


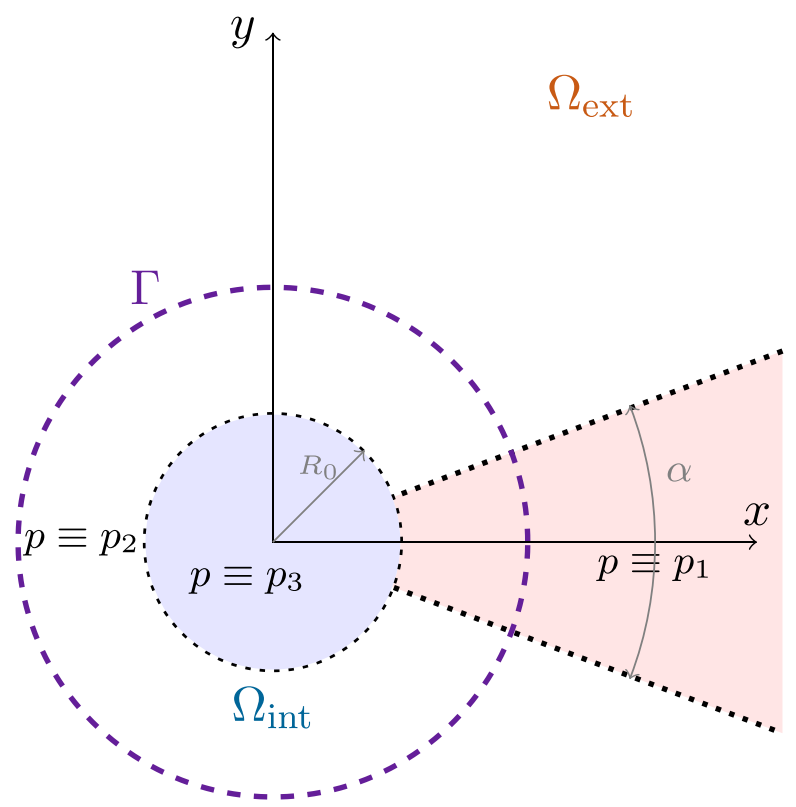

Fig. 3 Cross section of the domains for the examples in Figs. 4, 5, 6, and 7. The domains and potential functions $p$ are rotationally symmetric with respect to the $x$-axis

is rotationally symmetric with respect to the origin and can therefore be separated. The according resonances can be calculated semi-analytically by solving a non-linear scalar equation involving spherical Bessel and Hankel functions.

For all of our experiments we use fixed parameters $R_{0}=1, p_{1}=1, p_{2}=5$, and $p_{3}=10$. For the complex scaling we use $R=1.1$ (i.e., $\Omega_{\text {int }}=B_{R}, \Gamma=\partial B_{R}$, $\Omega_{\mathrm{ext}}=\mathbb{R}^{3} \backslash \overline{B_{R}}$ ), and varying parameters $\sigma$. For the discretization we use $N=20$ degrees of freedom in radial direction, a tetrahedral mesh of $\Omega_{\text {int }}$ with mesh size $h=0.3$ and polynomials of degree 3 .

Figure 4 shows resonances for $\alpha=0.6 \pi$ and two different complex scaling parameters $\sigma$. We observe spurious resonances in the vicinity of the sets $\{\omega \in \mathbb{C}: \Im(\omega \sigma)=$ $0\}$. This has to be expected from the results with homogeneous exterior domains in $[6,21,26]$. These resonances are a discretization of an essential spectrum. Moreover, it can be seen that the spurious resonances with in absolute values larger imaginary part move away from this axis. Probably, this effect is generated by discretization errors of the interior domain (see, e.g., [26]). More important is the fact that the resonances with $\Im(\omega \sigma)>0$ are independent of $\sigma$ and in the vicinity of the reference resonances for the homogeneous exterior domain.

In Fig. 5 cross-sections of selected resonance functions are given. They are in the vicinity of a resonance to the homogeneous exterior domain, i.e., $\alpha=0$. The multiplicity of the resonance in the homogeneous case is three, since the restrictions of the resonance functions on $\Gamma$ are spherical harmonics with index one. Due to the inhomogeneous exterior domain, this resonance is split into two perturbed resonances, one with multiplicity one (Fig. 5a) and the other (Fig. 5b) with multiplicity two. This 


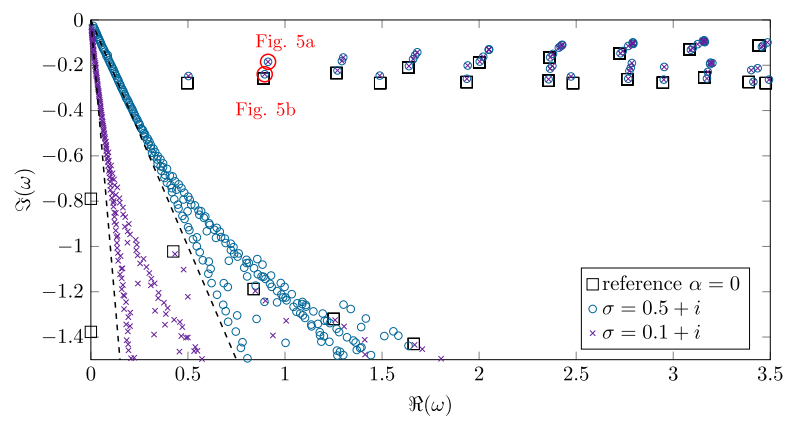

Fig. 4 Example with inhomogeneous exterior (cf. Fig. 3) with $\alpha=0.6 \pi, p_{1}=1, p_{2}=5, p_{3}=10$ and different parameters $\sigma$. The dashed lines mark the regions in the complex plane where $\Im(\omega \sigma)=0$ and therefore where we expect the essential spectra. Note that the reference resonances are for the separable problem with $\alpha=0$ and give merely an indication where we expect correct resonances

is due to the fact that the rotational symmetry of the domain with respect to the $x$-axis is preserved (cf. Fig. 3).

In Figs. 6 and 7 we studied the transformation of resonances from the reference configuration $\alpha=0$ to the other reference configuration $\alpha=2 \pi$. The resonances in Figs. $6 \mathrm{~b}$ and 7 correspond to spherical harmonics of index one.

\subsubsection{Radial and surface inhomogeneities}

In this subsection we approximate the resonances of Problem 2.1 on

$$
\Omega_{\mathrm{ext}}:=\Omega:=\mathbb{R}^{3} \backslash B_{1}(0)=\left\{\mathbf{x} \in \mathbb{R}^{3}:\|\mathbf{x}\|>1\right\}
$$

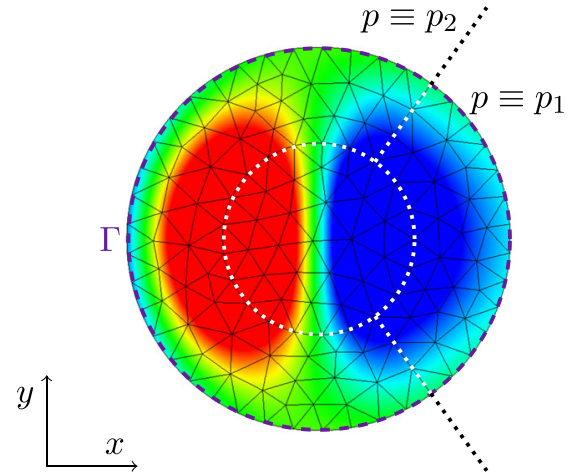

(a) $\omega \approx 0.91273-0.18479 i$

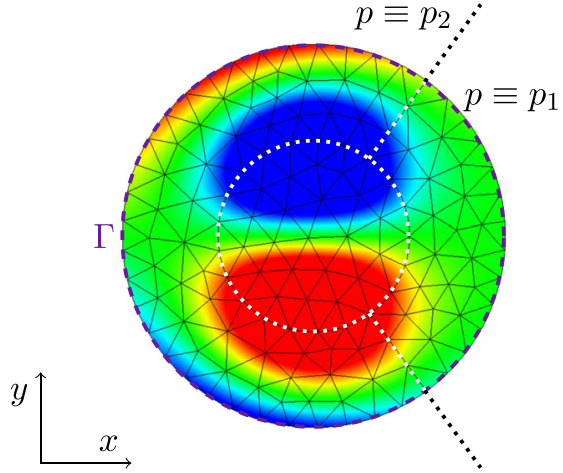

(b) $\omega \approx 0.89549-0.23975 i$

Fig. 5 Cross section of selected resonance functions from the experiment from Fig. 4. Note that, differing from Fig. 4, for a better visualization, $R=2$ was chosen and depicted are only the resonance functions in $\Omega_{\text {int }}$ 


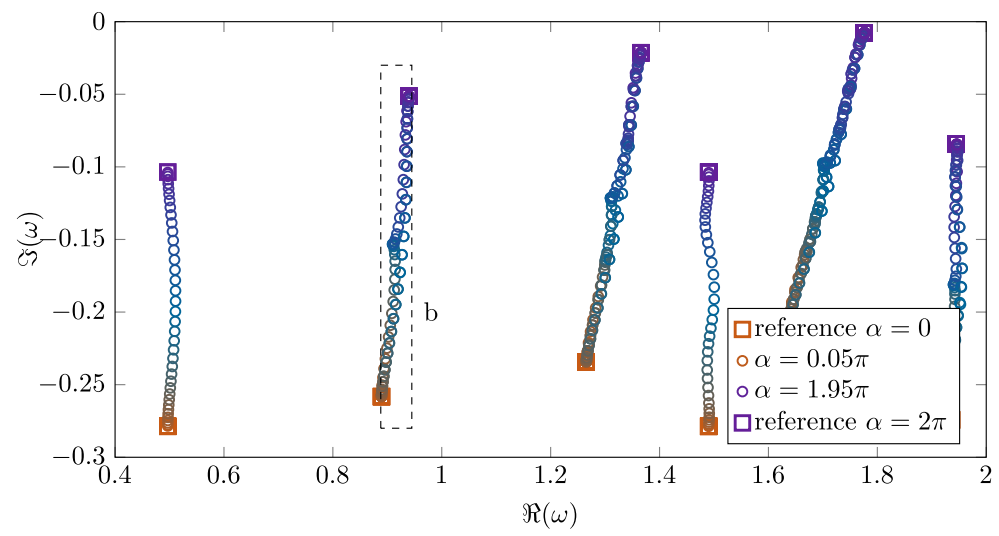

(a) dependency of resonances on the angle $\alpha$

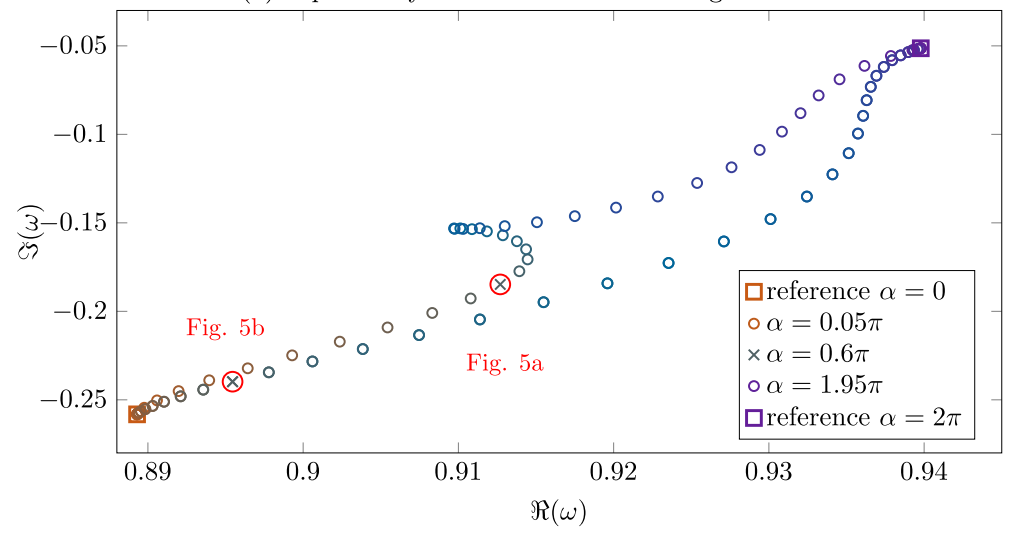

(b) close-up of (a)

Fig. 6 Example with inhomogeneous exterior (cf. Fig. 3) with $\sigma=0.3+i, p_{1}=1, p_{2}=5, p_{3}=10$ and different parameters $\alpha$. Note that the resonances on the branch with smaller imaginary part have multiplicity two

for potentials $p(\mathbf{x}(\xi, \hat{\mathrm{x}})):=\hat{p}(\hat{\mathrm{x}}) \tilde{p}(\xi)$ with functions $\hat{p}: \Gamma \rightarrow \mathbb{R}, \tilde{p}: \mathbb{R}_{\geq 0} \rightarrow \mathbb{R}$ given by

$$
\tilde{p}(\xi):=1+\tilde{\epsilon} \frac{(1+\xi)^{2}}{1+(1+\xi)^{4}}, \quad \hat{p}(\hat{\mathrm{x}}(x, y, z)):=1+\hat{\epsilon} z, \quad \xi \geq 0, \hat{\mathrm{x}} \in \Gamma
$$

for parameters $\tilde{\epsilon} \geq 0$ and $\hat{\epsilon} \in[0,1)$. For $\hat{\epsilon}=0$ we are in the situation of rotationally invariant potential functions. These problems can be separated into one-dimensional Bessel-like problems (cf. Section 2.4). If we assume in addition $\tilde{\epsilon}=0$, then we have the situation of Sections 6.1 and 2.2 with analytically known reference resonances. All three-dimensional experiments use the parameters $N=10$, finite element mesh size $h=0.3$ and polynomial degree $p=4$. For the separated one-dimensional 


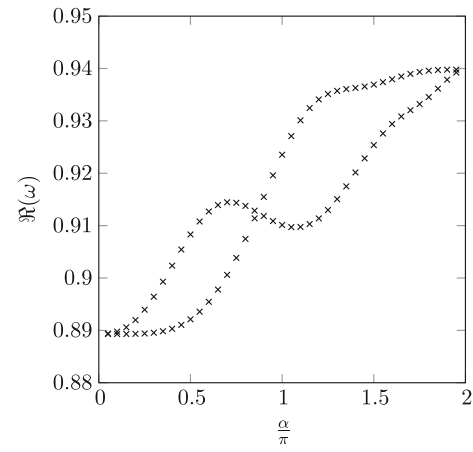

(a) Real parts

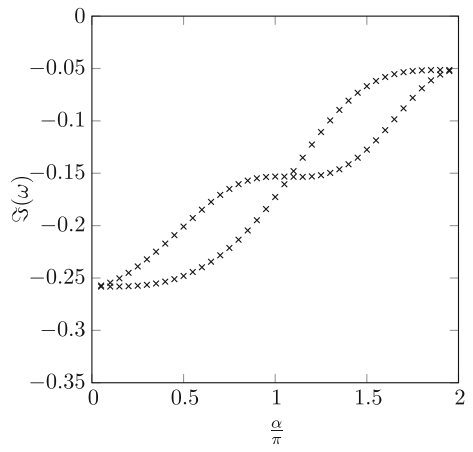

(b) Imaginary parts

Fig. 7 Real and imaginary parts of selected resonances from the experiment in Fig. 6

examples we used $N=200$. In this example we refrain from a presentation of results with different scaling constants $\sigma$. We merely note that the following results are in principle independent of the choice of $\sigma$ as long as $\Im(\omega \sigma)>0$. Note that the poles of the complex continuation of $\tilde{p}$ have negative real parts. Thus any choice of scaling parameter $\sigma$ with positive real and imaginary part leads to the correct complex scaling radiation condition, see Section 2.4. Of course, the approximation quality depends on $\sigma$.

In Fig. 8 we fixed $\hat{\epsilon}=0$ and use $\tilde{\epsilon} \in[0,1.5]$. The analytically given resonances for $\tilde{\epsilon}=0$ are marked by squares. Moreover, we solved the separated problems and the full three-dimensional problems. For larger values of $\tilde{\epsilon}$ the eigenvalues move

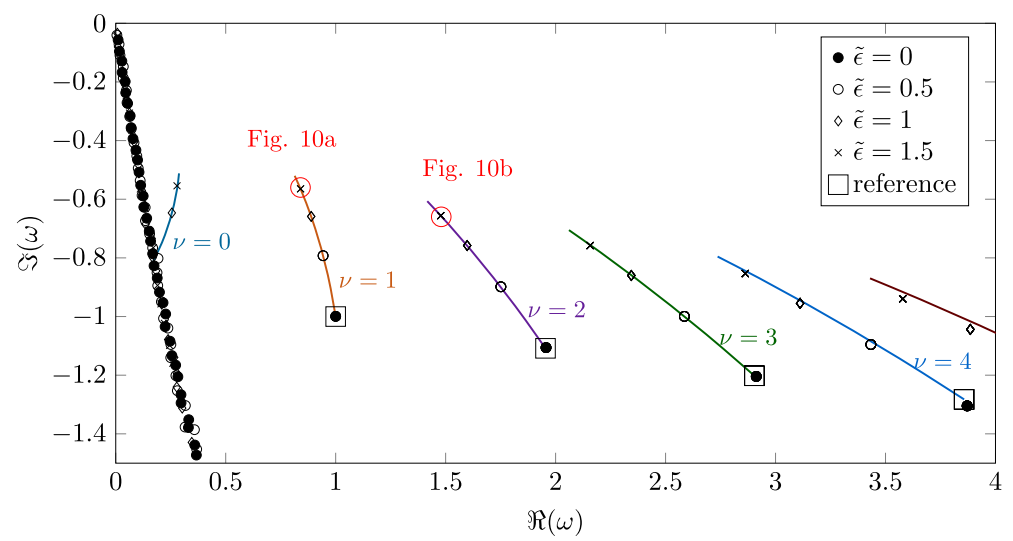

Fig. 8 Inhomogeneous exterior problem with radial inhomogeneity for discretization parameters $h=$ $0.3, \sigma=1+5 i, N=10$. The lines mark the locations of resonances for a given Hankel index $v$ and varying $\tilde{\epsilon}$, obtained by solving the separated problem, whereas the marks are eigenvalues calculated by solving the full three-dimensional problem. The reference resonances are the semi-analytically computed zeros of the derivatives of the spherical Hankel functions of the first kind 

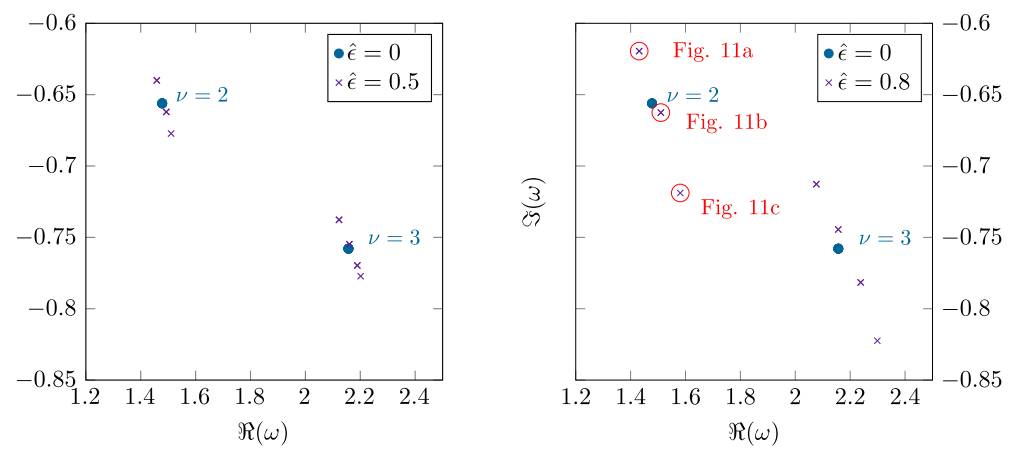

Fig. 9 Inhomogeneous exterior problem with $\tilde{\epsilon}=1.5$ and variable $\hat{\epsilon}$. Although this is a three-dimensional simulation, the given indices $v$ refer to the corresponding Hankel indices for the separated problem for $\hat{\epsilon}=0$

closer to the real axis. The approximated eigenvalues of the full three-dimensional simulation show a good agreement with the ones of the separated problem. The resonances located close to the negative imaginary axis in Fig. 8 are again part of the discretization of the essential spectrum.

Figure 9 shows resonances of the same problem but with $\hat{\epsilon}>0$. This problem is not separable any more. Thus only a three-dimensional simulation is possible. Due to the disturbed symmetry, the multiple eigenvalues fan out.

Figures 10 and 11 show selected resonance functions corresponding to the previously approximated resonances. To visualize the resonance functions, $\Omega_{\text {int }}=$ $B_{1.8}(0) \backslash \overline{B_{1}(0)}$ was chosen here. In Fig. $10 \hat{\varepsilon}$ is zero, i.e., we have a rotationally invariant problem with radial inhomogeneity. In Fig. 11 the problem is not rotational invariant leading to resonance functions with perturbed symmetry.

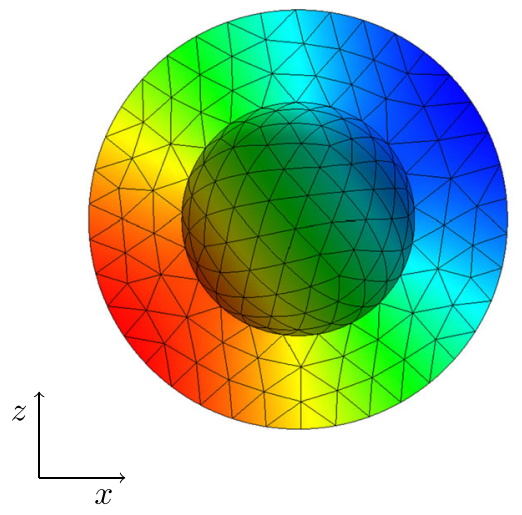

(a) $\omega \approx 0.8404-0.5645 i, \nu=1$

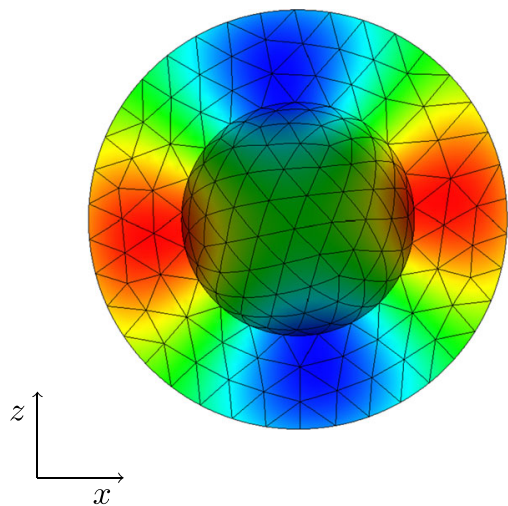

(b) $\omega \approx 1.4780-0.6561 i, \nu=2$

Fig. 10 Resonance functions corresponding to eigenvalues from Figs. 8 and 9 with $\tilde{\epsilon}=1.5$ and $\hat{\varepsilon}=0$. The given indices $v$ refer to the corresponding Hankel indices in the separated case 


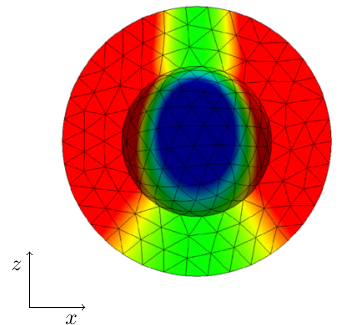

(a) $\omega \approx 1.4311-0.6194 i$

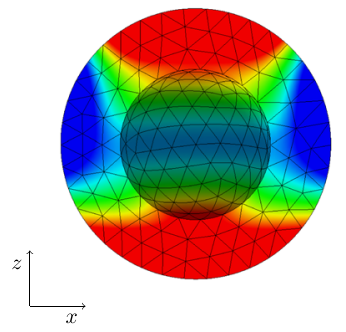

(b) $\omega \approx 1.5101-0.6625 i$

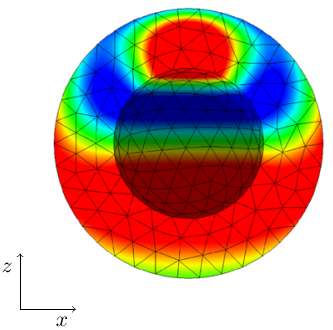

(c) $\omega \approx 1.5809-0.7188 i$

Fig. 11 Resonance functions corresponding to eigenvalues from Fig. 9 with $\tilde{\varepsilon}=1.5, \hat{\epsilon}=0.5$

\section{Conclusion}

In summary we can say that complex-scaled infinite elements are a very effective method for scalar resonance problems. They are more efficient compared to a standard PML due to their super-algebraic convergence.

Using continuous perturbations of well-established reference configurations, the numerical results indicate that complex-scaled infinite elements are well-suited for problems with inhomogeneous exterior domains. The convergence analysis covers these problems. Nevertheless, a rigorous derivation of radiation conditions in these situations, as well as the general applicability of complex scaling methods to these problems, remains an open question.

Funding Open access funding provided by TU Wien (TUW). This study was funded by the Austrian Science Fund (FWF): P26252.

\section{Declarations}

Conflict of interest The authors declare no competing interests.

Open Access This article is licensed under a Creative Commons Attribution 4.0 International License, which permits use, sharing, adaptation, distribution and reproduction in any medium or format, as long as you give appropriate credit to the original author(s) and the source, provide a link to the Creative Commons licence, and indicate if changes were made. The images or other third party material in this article are included in the article's Creative Commons licence, unless indicated otherwise in a credit line to the material. If material is not included in the article's Creative Commons licence and your intended use is not permitted by statutory regulation or exceeds the permitted use, you will need to obtain permission directly from the copyright holder. To view a copy of this licence, visit http://creativecommons.org/licenses/by/4.0/.

\section{References}

1. Abramowitz, M., Stegun, I.A.: Handbook of Mathematical Functions with Formulas, Graphs, and Mathematical Tables National Bureau of Standards Applied Mathematics Series, vol. 55. For sale by the Superintendent of Documents, U.S. Government Printing Office, Washington, D.C (1964)

2. Babuška, I., Osborn, J.: Eigenvalue Problems. In: Handbook of Numerical Analysis, Vol. II, Handb. Numer. Anal., II, pp. 641-787. North-Holland, Amsterdam (1991) 
3. Berenger, J.P.: A perfectly matched layer for the absorption of electromagnetic waves. J. Comput. Phys. 114(2), 185-200 (1994)

4. Bermúdez, A., Hervella-Nieto, L., Prieto, A., Rodríguez, R.: An exact bounded perfectly matched layer for time-harmonic scattering problems. SIAM J. Sci. Comput. 30(1), 312-338 (2007). https://doi.org/10.1137/060670912

5. Bramble, J.H., Pasciak, J.E.: Analysis of a Cartesian PML approximation to acoustic scattering problems in $\mathbb{R}^{2}$ and $\mathbb{R}^{3}$. J. Comput. Appl. Math. 247, 209-230 (2013). https://doi.org/10.1016/j.cam. 2012.12.022

6. Collino, F., Monk, P.: The perfectly matched layer in curvilinear coordinates. SIAM J. Sci. Comput. 19(6), 2061-2090 (1998). (electronic)

7. Colton, D., Kress, R. Inverse Acoustic and Electromagnetic Scattering Theory Applied Mathematical Sciences, second edn, vol. 93. Springer-Verlag, Berlin (1998)

8. Dziuk, G., Elliott, C.M.: Finite element methods for surface PDEs. Acta Numer. 22, 289-396 (2013). https://doi.org/10.1017/S0962492913000056

9. Halla, M.: Convergence of hardy space infinite elements for helmholtz scattering and resonance problems. SIAM Journal on Numerical Analysis 54(3), 1385-1400 (2016). https://doi.org/10.1137/ $15 \mathrm{M} 1011755$

10. Halla, M.: Analysis of radial complex scaling methods for scalar resonance problems in open systems. PhD. thesis, TU Vienna Wien (2019)

11. Halla, M.: Galerkin approximation of holomorphic eigenvalue problems: weak T-coercivity and Tcompatibility. Numer. Math. (148), 387-407. https://doi.org/10.1007/s00211-021-01205-8 (2021)

12. Halla, M.: Analysis of radial complex scaling methods: scalar resonance problems. SIAM J. Numer. Anal. 59, 2054-2074 (2021). https://doi.org/10.1137/20M1354234

13. Hislop, P.D., Sigal, I.M.: Introduction to spectral theory, Applied Mathematical Sciences, vol. 113. Springer-Verlag, New York. https://doi.org/10.1007/978-1-4612-0741-2, With applications to Schrödinger operators (1996)

14. Hohage, T., Nannen, L.: Hardy space infinite elements for scattering and resonance problems. SIAM J. Numer. Anal. 47(2), 972-996 (2009)

15. Hohage, T., Nannen, L.: Convergence of infinite element methods for scalar waveguide problems. BIT Numerical Mathematics 55(1), 215-254 (2015). https://doi.org/10.1007/s10543-014-0525-X

16. Hohage, T., Schmidt, F., Zschiedrich, L.: Solving time-harmonic scattering problems based on the pole condition. I. Theory. SIAM J. Math. Anal. 35(1), 183-210 (2003)

17. Hohage, T., Schmidt, F., Zschiedrich, L.: Solving time-harmonic scattering problems based on the pole condition. II. Convergence of the PML method. SIAM J. Math. Anal. 35(3), 547-560 (2003)

18. Karma, O.: Approximation in eigenvalue problems for holomorphic Fredholm operator functions. I. Numer. Funct. Anal. Optim. 17(3-4), 365-387 (1996). https://doi.org/10.1080/01630569608816699

19. Karma, O.: Approximation in eigenvalue problems for holomorphic Fredholm operator functions. II. (Convergence rate). Numer. Funct. Anal. Optim 17(3-4), 389-408 (1996). https://doi.org/10.1080/ 01630569608816700

20. Kato, T.: Perturbation theory for linear operators. Classics in Mathematics. Springer-Verlag, Berlin (1995). Reprint of the 1980 edition

21. Kim, S., Pasciak, J.E.: The computation of resonances in open systems using a perfectly matched layer. Math. Comp. 78(267), 1375-1398 (2009). https://doi.org/10.1090/S0025-5718-09-02227-3

22. Kim, S., Pasciak, J.E.: Analysis of the spectrum of a Cartesian perfectly matched layer (PML) approximation to acoustic scattering problems. J. Math. Anal. Appl. 361(2), 420-430 (2010). https://doi.org/10.1016/j.jmaa.2009.07.024

23. Lassas, M., Somersalo, E.: On the existence and the convergence of the solution of the pml equations. Computing 60, 229-241 (1998)

24. Moiseyev, N.: Quantum theory of resonances: Calculating energies, width and cross-sections by complex scaling. Physics reports 302, 211-293 (1998)

25. Nannen, L., Schädle, A.: Hardy space infinite elements for Helmholtz-type problems with unbounded inhomogeneities. Wave Motion 48(2), 116-129 (2010). https://doi.org/10.1016/j.wavemoti.2010.09. 004

26. Nannen, L., Wess, M.: Computing scattering resonances using perfectly matched layers with frequency dependent scaling functions. BIT 58(2), 373-395 (2018). https://doi.org/10.1007/s10543018-0694-0 
27. Saad, Y.: Numerical methods for large eigenvalue problems, Classics in Applied Mathematics, vol. 66. Society for Industrial and Applied Mathematics (SIAM), Philadelphia, PA. https://doi.org/10. 1137/1.9781611970739.ch1. Revised edition of the 1992 original [ 1177405] (2011)

28. Schöberl, J.: Netgen - an advancing front 2d/3d-mesh generator based on abstract rules. Comput. Visual. Sci 1, 41-52 (1997)

29. Schöberl, J.: C++11 Implementation of Finite Elements in Ngsolve. Preprint 30/2014. Institute for Analysis and Scientific Computing, TU Wien (2014)

30. Shen, J., Tang, T., Wang, L.L.: Spectral methods, Springer Series in Computational Mathematics, vol. 41. Springer, Heidelberg. https://doi.org/10.1007/978-3-540-71041-7. Algorithms, analysis and applications (2011)

31. Steinbach, O., Unger, G.: Convergence analysis of a Galerkin boundary element method for the Dirichlet Laplacian eigenvalue problem. SIAM J. Numer. Anal. 50, 710-728 (2012)

32. Wess, M.: Frequency-dependent complex-scaled infinite elements for exterior helmholtz resonance problems. PhD. thesis, TU Vienna Wien (2020)

Publisher's note Springer Nature remains neutral with regard to jurisdictional claims in published maps and institutional affiliations. 